\title{
Model-Based Quantitative Evaluation of Repair Procedures in Gas Distribution Networks
}

\author{
MARCO BIAGI, LAURA CARNEVALI, FABIO TARANI, and ENRICO VICARIO, \\ University of Florence
}

\begin{abstract}
We propose an approach for assessing the impact of multi-phased repair procedures on gas distribution networks, capturing load profiles that can depend on time for different classes of users, suspension of activities during non-working hours, and random execution times depending on topological, physical, and geographical characteristics of the network. The problem is characterized through a semi-formal specification based on artifacts of the Systems Modeling Language (SysML), which is then translated into a formal model based on stochastic time Petri nets. The solution method interleaves fluid-dynamic analysis of the gas behavior and stochastic analysis of the time spent in the repair process, decoupling complexities and making stochastic analysis almost insensitive to the network size and topology. Hence, our approach turns out to be applicable to real scale cases, notably computing the optimal time of day to start the repair procedure. Moreover, by encompassing general (non-Markovian) distributions, the approach enables effective fitting of durations.
\end{abstract}

CCS Concepts: • General and reference $\rightarrow$ Reliability; Performance; • Computer systems organization $\rightarrow$ Maintainability and maintenance;

Additional Key Words and Phrases: Gas distribution networks, repair procedures, performability measures, low pressure risk, stochastic durations

\section{ACM Reference format:}

Marco Biagi, Laura Carnevali, Fabio Tarani, and Enrico Vicario. 2018. Model-Based Quantitative Evaluation of Repair Procedures in Gas Distribution Networks. ACM Trans. Cyber-Phys. Syst. 3, 2, Article 19 (December 2018), 26 pages.

https://doi.org/10.1145/3284037

\section{INTRODUCTION}

\subsection{Motivation}

Gas networks are Cyber-Physical Systems (CPSs) coupling (physical) fluid-dynamic processes with (cyber) management procedures. Due to the increasing interdependence with other critical infrastructures, notably electric power systems, performance and reliability of gas networks have a relevant impact on industry, economy, and security. In this context, quantitative evaluation methods may contribute to improve the maturity level of operation and maintenance processes, also leveraging the ongoing spread of smart devices for monitoring and control, and the consequent increasing availability of data in the field (Abbatantuono et al. 2016).

Authors' addresses: M. Biagi, L. Carnevali, F. Tarani, and E. Vicario, via di Santa Marta 3, 50139 Firenze (FI), Italy; emails: \{marco.biagi, laura.carnevali, fabio.tarani, enrico.vicario\}@unifi.it.

Permission to make digital or hard copies of part or all of this work for personal or classroom use is granted without fee provided that copies are not made or distributed for profit or commercial advantage and that copies bear this notice and the full citation on the first page. Copyrights for third-party components of this work must be honored. For all other uses, contact the Owner/Author.

(C) 2018 Copyright held by the owner/author(s).

2378-962X/2018/12-ART19 \$15.00

https://doi.org/10.1145/3284037 


\subsection{Contribution}

We propose a comprehensive methodology for performability evaluation of a gas distribution network during a repair procedure. To the best of our knowledge, this addresses for the first time three different elements of complexity in the analysis of a repair procedure: non-Markovian duration of repair activities, suspension of operations during non-working hours, and time-varying load profiles (Section 1.3). Specifically, we propose a semi-formal specification based on artifacts of SysML to characterize the hybrid behavior of the network, which couples the continuous dynamics of fluid physics with the discrete dynamics of state changes induced by network management (Sections 2.1 and 2.2). Then, we derive a stochastic Petri net model of the procedure from an Activity Diagram connected with State Charts of state-dependent resources (Section 2.3), and we interleave fluiddynamic analysis of the network with stochastic analysis of the procedure, evaluating (i) the risk that pressure is not sufficient for demand during the procedure and (ii) the Cumulative Distribution Function (CDF) of the completion time of each phase of the procedure (Section 3). We validate the approach on the case of a real network (Szoplik 2012) with actual load profiles, and we perform a sensitivity analysis with respect to the number of steps used to represent pressure regulation, the temporal discretization considered for demand variations, and the stochastic distribution assumed for the execution times of repair activities (Section 4). Experimental results prove the feasibility of the approach, pointing out the substantial invariance of computational complexity with respect to network size and topology, and notably supporting the selection of the optimal time of day for the maintenance process to start. Finally, applicability of the approach and further steps enabled by the results are discussed (Section 5).

\subsection{Related Works}

1.3.1 Operation and Maintenance of Gas Networks. Several works on quantitative evaluation of availability of gas networks focus on transmission infrastructures, which convey gas at high pressure from entry terminals to gas distribution networks or power stations. A Markov model of a gas compression station and the surrounding pipeline network is presented in Praks et al. (2007), considering changes of gas demand over time. In the ProGasNet project (Praks et al. 2014), Monte Carlo simulation and fault tree analysis are used to evaluate reliability and security issues of a gas transmission network based on failure probabilities of components. Historical data are used in Vianello and Maschio (2014) to perform quantitative risk assessment of pipelines in the Italian gas transmission network, considering local and social risks. In Wang and Duncan (2014), incident data on U.S. gas transmission pipelines support the assessment of causes and consequences of failures with respect to property damage, fatality or injury rate, and volume of gas released.

Gas distribution networks operate at medium to low pressure, posing different challenges due to a more densely connected topology, a higher variability in load and location of customers, and no gas storage capacity in pipes. While the proposed approach integrates fluid-dynamic analysis of the network with stochastic analysis of a maintenance procedure, a large part of the literature focuses on fluid-dynamic analysis only, typically to assess flow rates and pressures (González et al. 2009; Szoplik 2012), or addresses optimization of operation disregarding uncertainty on network reliability and maintenance duration, notably to mitigate system volatility (Zavala 2014), to optimize design decisions and operating flows (Li et al. 2011), and to favor integration in multi-carrier systems combining provisioning of electric and gas power (Li et al. 2008; Qadrdan et al. 2014).

Some works address demand forecast exploiting weather, economic, and consumption data (Soldo 2012), especially to detect anomalies in gas usage through a nearest-neighbor algorithm and a local regression analysis (Baldacci et al. 2016), to predict gas demand of residential and commercial users by training artificial neural networks with temperature and calendar data (Szoplik 2015), 
and to forecast the annual gas demand in Europe through a structural time-series model considering income, gas price, and energy demand trend (Dilaver et al. 2014). As a major difference, these approaches use load prediction to support short-term monitoring of the network for security reasons and to optimize gas procurement in the medium-long term, while the proposed approach leverages load data to assess network performability and plan maintenance interventions.

Stochastic modeling is applied for performability evaluation of a gas distribution network, notably to compute average measures of interruption rate and outage time under the assumption of deterministic repair times (Helseth and Holen 2006), to derive the probability that all end-users are connected to the distribution node based on the failure probability of network components (Pino et al. 2016), and to sort pipeline sections into risk categories (Brito et al. 2010). Yet, very few works allow for uncertainty in repair duration. In Esposito et al. (2013), performance of the gas distribution network in L'Aquila, Italy, after the 2009 earthquake is assessed, reporting observed minimum, maximum, and average repair times. Preliminary results on the performability evaluation of a small-sized network after a pipe break are discussed in Carnevali et al. (2014), considering activities with stochastic duration while referring to a simplified representation of demand variations and disregarding the usual suspension of repair operations during non-working hours.

1.3.2 Semi-Formal Specification of Repair Procedures. Specification of the repair procedure faces a twofold methodological requirement: for the derivation of measures of interest, the overall cyber-physical process must be represented in a formal model amenable to quantitative evaluation; however, acceptance in the context of use demands a notation that can meet the standards of the industrial practice. Reconciliation of the two perspectives has been faced through automated model transformation in a wide range of literature (Balsamo et al. 2004; Koziolek 2010), targeted to support the engineering of non-functional requirements, mainly concerned with performance or dependability.

A large part of this literature develops on semi-formal artifacts of the Unified Modeling Language (UML), often extended by the profile for Modeling and Analysis of Real-Time Embedded systems (MARTE) (Bernardi et al. 2013; Object Management Group 2009) or by the Systems Modeling Language (SysML) (Object Management Group 2017). In particular, in SysML the bias toward computing-oriented concepts is reduced to better encompass systems involving physical components, personnel, and procedures. In both cases, either with UML or SysML, the specification consists of multiple cross-connected source models (Mens and Van Gorp 2006), with some emerging methodological patterns: Use Case Diagrams provide a high-level functional identification of scenarios; the detailed flow of execution of each scenario is represented by Sequence Diagrams or Activity Diagrams or State Charts; the structural perspective is typically represented by Component Diagrams and Deployment Diagrams in UML, or by Block Diagrams in SysML. In addition, SysML provides a Requirements Diagram or an equivalent Requirements Table to organize and connect functional, structural, and quality requirements, and a Parametric Diagram to specify constraints and equations characterizing other modeling components. The approach presented in this article effectively applies SysML modeling principles to specify repair procedures for gas distribution networks, providing insight to apply the methodology beyond this specific application context.

1.3.3 Formal Specification of Repair Procedures. Various approaches have been proposed to increase the level of rigor of UML or SysML specifications so as to make them amenable to quantitative evaluation through automated translation into models based on queuing networks, stochastic process algebras, stochastic Petri nets, and stochastic processes (Balsamo et al. 2004). Most works restrain durations to be (memoryless) exponential random variables (EXP), using formalisms such as Generalized Stochastic Petri Nets (GSPNs) (Bernardi et al. 2002) or Performance Evaluation 
Process Algebra (PEPA) (Tribastone and Gilmore 2008), whose underlying stochastic process is a Continuous Time Markov Chain (CTMC) and can be solved through consolidated techniques.

Only few works have addressed the case of generally distributed durations (GEN), possibly constrained to have a bounded support, which afford a higher solution complexity to obtain more valid expressivity in representation of non-functional system characteristics. In Lindemann et al. (2002), UML State Charts or Activity Diagrams are extended with EXP and deterministic (DET) delays and then mapped onto a subclass of Generalized Semi-Markov Process (GSMP) amenable to numerical solution through the method of equidistant sampling (Lindemann and Thümmler 1999). In Bernardi et al. (2011), single steps of a Sequence Diagram are extended with min-max constraints on durations, and efficient techniques are used to over-approximate the completion time support; a measure of likelihood for the completion time to break a deadline is then derived under the assumption that all durations are equiprobable, i.e., that the distribution of the completion time is uniform over the approximated support. In Homm and German (2016), states in parallel regions of a State Chart are associated with GEN sojourn times and synchronized with operators that permit a compositional solution through hierarchical composition of Semi Markov Processes (SMPs). The model expressivity is significantly extended in Biagi et al. (2018) allowing system evolution to be dependent on the exited region. The approach proposed in this article translates the Activity Diagram of the procedure and the State Charts of the network and the personnel into a class of stochastic time Petri nets, extending previous results of the literature by representing GEN durations of multiple concurrent activities, DET durations of time zones capturing personnel working hours and load demand profiles, and suspension/resumption of activities that can be performed only during working hours according to the semantics of Preemptive ReSume (Bobbio et al. 2000).

\section{CHARACTERIZATION OF THE APPLICATION CONTEXT}

We outline the problem of performability of a gas distribution network during a maintenance procedure (Section 2.1); we then characterize it through a semi-formal specification based on artifacts of SysML (Section 2.2), and we finally derive a formal stochastic model (Section 2.3).

\subsection{Problem Description}

In a gas distribution network, pipes connect regulating stations (supply nodes) to users (load nodes). While pressure at each supply node is controlled by a regulator device, pressures at load nodes depend on the network topology and on the flow rates withdrawn by users, with emerging daily patterns. In normal operation, supply pressures are kept at constant values such that pressure at each load node is above a minimum, to avoid device malfunction and guarantee the quality of service, and below a maximum (significantly larger than the minimum), for safety reasons, economy of operation, and reduced environmental impact of leakage.

During maintenance, the network topology is changed to isolate and restore affected components, making the network behavior deviate from nominal operation. The entity of deviation can be mitigated by regulating pressures at supply nodes. Since contingencies may occur at random times and maintenance steps take random durations, changes occur at stochastic time points, resulting in different completion times of procedure phases and in different exposure to the risk of incurring pressures too low with respect to safety and contractual constraints. Hence, key measures of performability of a repair procedure are the probability distribution of completion times of procedure phases and the risk that, during the procedure, pressure becomes insufficient for the actual demand.

In this work, we describe a method for evaluating both these measures, focusing on the procedure for repairing a pipe (Cagno et al. 2000) in a network with a single supply node. As discussed in Section 2.2.5, the method can be extended to encompass other maintenance operations and to 


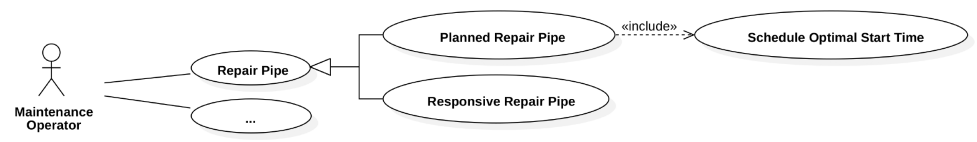

Fig. 1. Use Case Diagram identifying the scenario of pipe repair.

Table 1. Requirements in Tabular Form

\begin{tabular}{|c|l|}
\hline Use Case & \multicolumn{1}{c|}{ Requirements } \\
\hline \multirow{2}{*}{$\begin{array}{c}\text { Planned } \\
\text { Repair Pipe }\end{array}$} & $\begin{array}{l}\text { The procedure Completion Time must be }<36 \mathrm{~h} \text { in } 75 \% \text { of the cases, and } \\
<48 \mathrm{~h} \text { in } 99.99 \% \text { of the cases. }\end{array}$ \\
\cline { 2 - 2 } The Low-Pressure Risk at any time must be $<240 \mathrm{bar}^{1 / 2}$ on average. \\
\hline \multirow{2}{*}{$\begin{array}{l}\text { Responsive } \\
\text { Repair Pipe }\end{array}$} & $\begin{array}{l}\text { The procedure Completion Time must be }<24 \mathrm{~h} \text { in } 75 \% \text { of the cases, and } \\
<36 \mathrm{~h} \text { in } 99.99 \% \text { of the cases. }\end{array}$ \\
\cline { 2 - 3 } & The Low-Pressure Risk at any time must be $<360 \mathrm{bar}^{1 / 2}$ on average. \\
\hline
\end{tabular}

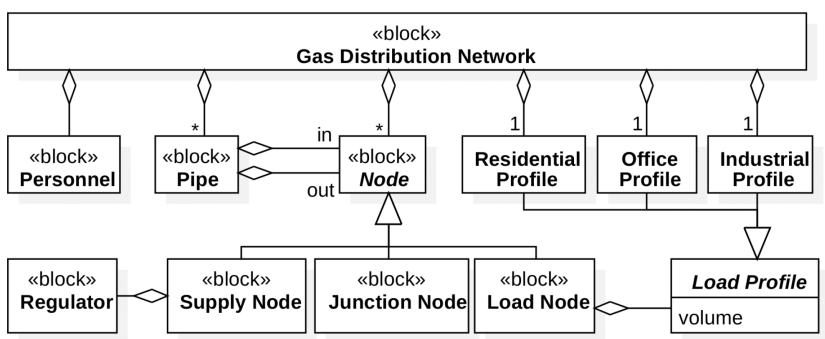

Fig. 2. Block Diagram description of resources involved in the operation of a gas distribution network.

accommodate multiple supply nodes. The model and the evaluation include activities that can be suspended and resumed according to working/overtime hours of personnel on field, in parallel to others that can be run concurrently without time limitations. Variation over time of gas demand is modeled by deterministic patterns, distinguishing residential, office, and industrial load, as in the practice of domain experts and institutional operators (e.g., the Regulatory Authority for Electricity and Gas (AEEGSI 2017) in Italy, one of the largest natural gas consuming countries in Europe (Deane et al. 2017)). While in reality the intensity of withdrawn flows varies with continuity, load patterns are discretized as stepwise functions to limit the number of different configurations for which fluid-dynamic analysis must be performed: experimental results of Section 4 show that a time granularity of $30 \mathrm{~min}$ is definitely sufficient to attain the accuracy requested for the context of use.

\subsection{Semi-Formal Specification}

We propose a specification where operation scenarios are identified by Use Case Diagrams (Figure 1) with requirements listed in a Requirements Table (Table 1); the structure of the system is described by a Block Diagram (Figure 2); the behavior of state-dependent Blocks is captured by a set of State Charts (Figure 3); the detailed procedure is specified by an Activity Diagram (Figure 4) connected with such State Charts. For the sake of space and readability, various details are omitted in the syntax of stereotypes and in the formalization of constraints in the form of a Parametric Diagram. Rather, emphasis is put on how artifacts are combined together in a way that can meet 


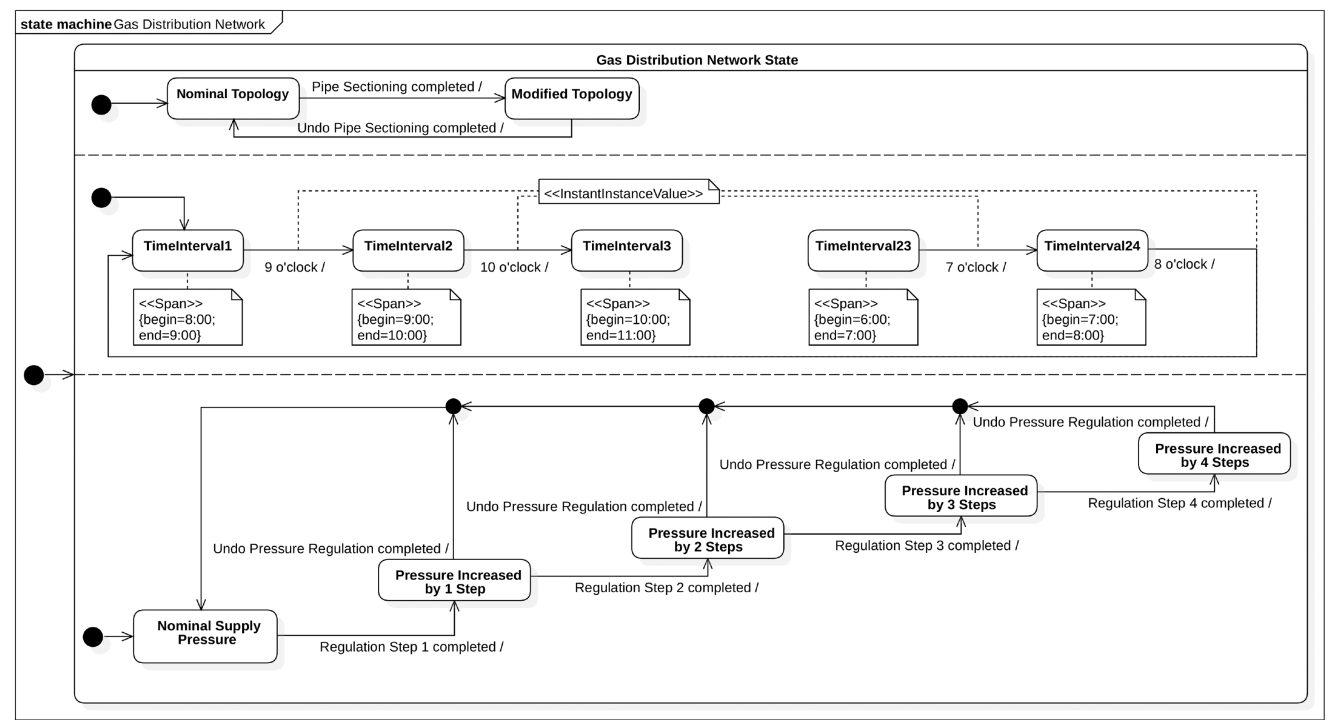

(a)

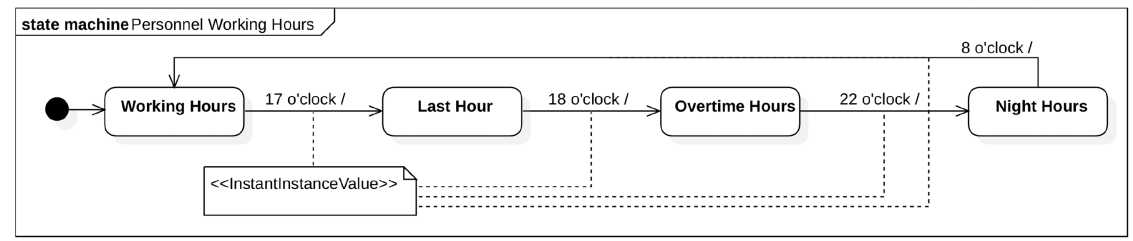

(b)

Fig. 3. State Charts of (a) the Gas Distribution Network behavior and (b) the Personnel working hours.

the practice of an industrial context and, at the same time, enable derivation of a formal stochastic model, as later developed in Section 2.3.

2.2.1 Use Case Diagrams. Figure 1 shows a fragment of the Use Case Diagram identifying the scenario of pipe repair, which might be extended further to specify other repair actions.

2.2.2 Requirements Table. Table 1 lists requirements and associates them with use case scenarios of Figure 1 (to keep notation light, we avoid specifying an identifier, a supplier, etc., for each requirement). In the specification, the quantities Completion Time and Low-Pressure Risk are identified as parametric values which will be the object of the evaluation of Section 3 (the Low-Pressure Risk is computed according to Equations (5) and (6) with $P_{\min }=18.0 \mathrm{mbar}$, $P_{\text {thr }}=20.0 \mathrm{mbar}$, and $\alpha=1.5$ ).

2.2.3 Block Diagram. In Figure 2, the static structure of resources involved in the repair process is specified as a Block Diagram. Specifically, the gas distribution network, its structural components (i.e., Pipes and Nodes of different types), and the Personnel that composes the working team are classified as resources by the SysML stereotype $<<$ block $>>$. Following the practice of gas networks operation, each Load Node is associated with a specific volume, but the profile over time is assumed to be common to all nodes of the same type (Residential, office, or Industrial). 


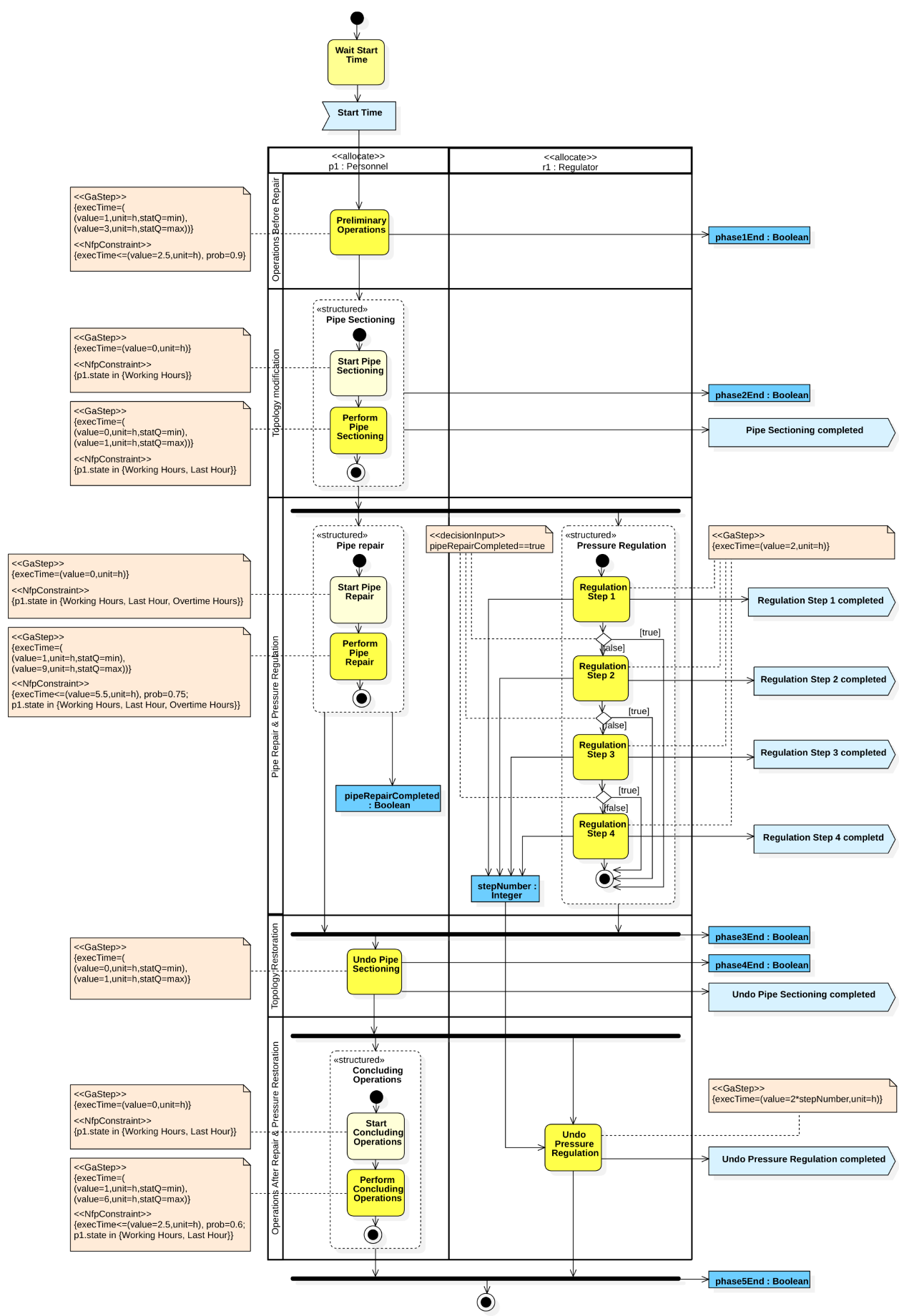

Fig. 4. Activity Diagram of the repair procedure. 
2.2.4 State Charts. Resources that behave differently depending on some internal state are characterized as Extended Finite State Machines using the notation of State Charts. The State Chart in Figure 3(a) uses a composite state with three nested sub-states to capture the different fluid-dynamic response of the Gas Distribution Network under (i) the different states of connection of the Pipe interested by the repair operation; (ii) the different flows withdrawn by Load Nodes at different times of day; and (iii) the different pressure levels applied by the Regulator at the Supply Node.

(1) During the maintenance procedure, the affected pipe is disconnected from the network to guarantee its safe repair, and it is then reconnected when repair is completed.

(2) Flows withdrawn by Load Nodes change periodically according to a daily Load Profile, as mentioned in Sections 2.1 and 2.2.3. The State Chart of Figure 3(a) represents the passage of daily time with the granularity of $1 \mathrm{~h}$, using the MARTE stereotypes $<<$ InstantInstanceValue $>>$ and $<<$ Span $>>$ to model the striking of the hours and the time intervals between two consecutive times of day, respectively. Note that the MARTE stereotype $<<$ ClockResource $>>$ might have been used to model an absolute time of day clock, generating an asynchronous message every hour. Yet, this choice was avoided to keep notation light.

(3) The supply pressure is raised during pipe repair to mitigate deviation from nominal operation, and it is restored to its initial value, at the same rate of increase, when repair is over or when all online nodes (i.e., connected to supply) receive sufficient pressure. Regulation is slow and takes a time depending on the Regulator characteristics, the pressure increase $\Delta P$ guaranteeing sufficient pressure for all online nodes, and the duration of pipe repair (regulation is stopped if it is ongoing when repair is completed). Hence, durations of pressure increase and decrease are dependent random variables taking the same value.

For low pressure networks connected to end-users, the lower bound on gauge pressure not to be exceeded to avoid device malfunctioning is around 17-19 mbar, while the upper bound for safe operation is 40 mbar. During pressure regulation, the risk of violating the minimum pressure constraint at load nodes becomes relevant. Though the supply pressure is a continuous variable, in the solution method pressure increase is discretized in a finite number of steps, not only to limit the number of configurations for which fluid-dynamic analysis has to be performed (as for load discretization) but also to determine the time needed for pressure restoration. Experimental results presented in Section 4 show that 32 steps for pressure increase lead to sufficient accuracy in the evaluation of performability measures. To reduce the cluttering, the State Chart of Figure 3(a) shows four steps for pressure regulation, corresponding to an increase of $0.25 \Delta P, 0.5 \Delta P, 0.75 \Delta P$, and $\Delta P$, respectively.

Conversely, after regulation, pressure at load nodes does not generally exceed the maximum threshold, and thus pressure reduction is represented by a single step in Figure 3(a). Anyway, the case where pressure at some load node exceeds the maximum threshold could be easily accommodated in the approach through multiple steps for pressure reduction.

The State Chart in Figure 3(b) specifies the plan of working hours for the Personnel, again using the MARTE stereotype <<InstantInstanceValue $>>$ to model the striking of the hours. Specifically, working hours are from 8:00 to 18:00, but activities can continue further, up to 22:00, if pipe repair is ongoing, and cannot be suspended at all if preliminary operations are ongoing. The last working hour accounts for pipe sectioning, which cannot be started after 17:00. Any other schedule where suspension is bound to have a deterministic duration is fully supported by the proposed approach. 
Note that, in the methodological perspective, the notation of State Charts is here used to characterize the evolution of the state of a gas distribution network subject to maintenance across discrete locations, without capturing continuous variables (e.g., the time elapsed since the start of Repair Pipe, the pressure level reached by the Regulator of the Supply Node), which could be more conveniently specified through mathematic equations in the parametric part of the SysML model.

2.2.5 Activity Diagram. In Figure 4, the cyber perspective on the overall maintenance procedure is specified through an Activity Diagram. Vertical swimlanes are used to allocate actions to resources identified as blocks in the Block Diagram of Figure 2, as in the practice of SysML, while horizontal swimlanes are used to represent the procedure phases. Following Esposito et al. (2013), five phases are considered. Specifically, after preliminary operations (Operations Before Repair), the nearest valves located upstream of and downstream from the affected pipe are closed to allow repair and, if needed, other valves are opened to limit the number of offline nodes, i.e., not connected to supply (Topology Modification). During repair, the supply pressure is raised until all online nodes have sufficient pressure or the pipe has been repaired (Pipe Repair \& Pressure Regulation). As discussed in Section 2.2.4, regulation is discretized in a finite number of steps (four in Figures 3(a) and 4), and it is represented here as a structured activity with an action for each step. Finally, the original topology is restored (Topology Restoration), the supply pressure is lowered to its initial value, and concluding operations are performed (Operations After Repair \& Pressure Restoration). Note that the object node pipeRepairCompleted represents a Boolean variable that becomes true when Pipe Repair is over; the object node stepNumber accounts for an integer variable representing the number of steps completed during Pressure Regulation; $\forall N \in\{1,2, \ldots, 5\}$, the object node phaseNEnd models a Boolean variable that becomes true when the $N$-th phase of the procedure is completed; and, signals correspond to events in the State Chart of Figure 3(a), e.g., Pipe Sectioning completed.

Execution times of maintenance actions depend on network and load data (including the network topology), the characteristics of the failed pipe, the number of regulation steps, and the pressure increase $\Delta P$. Figure 4 shows data informally reported from the practice, thanks to the experience gained in a research collaboration with a company that provides utilities to gas distribution operators. Such data can be collected through devices already in use on the field, and are in the same order of magnitude as those presented in Esposito et al. (2013). Regulation steps take a deterministic time determined by $\Delta P$, the number of steps in the procedure model, and the (constant) increase rate of the Regulator. Conversely, the remaining activities have an execution time ranging in an interval [min, $\max$ ], which can be conveniently estimated by analogy from the long-term experience of involved personnel and may be associated with a probabilistic characterization, identifying a value $x \in(\min , \max )$ such that the execution time is lower than $x$ with probability $p$. The MARTE stereoptype GaStep is used to specify execution time intervals, e.g., each step of Pressure Regulation has an execution time of $2 \mathrm{~h}$, Undo Regulation has an execution time of $2 \cdot$ stepNumber $\mathrm{h}$, and Pipe Repair has an execution time supported over the interval $[1,9]$ h. The MARTE stereotype $\mathrm{NfpConstraint} \mathrm{is} \mathrm{used} \mathrm{to} \mathrm{represent} \mathrm{probabilistic} \mathrm{constraints} \mathrm{on} \mathrm{execution} \mathrm{times,} \mathrm{e.g.,} \mathrm{Pipe} \mathrm{Repair}$ has an execution time lower than $5.5 \mathrm{~h}$ in $75 \%$ of the cases.

Some maintenance actions can be started and executed by the Personnel according to working/overtime hours, and may be suspended during the night and resumed at 8:00 on the next day. In this case, a structured activity is used to distinguish the start of a maintenance action from its execution, using the MARTE stereotype $<<$ NfpConstraint $>>$ to specify Personnel hours. This is the case of Pipe Sectioning, Pipe Repair, and Concluding Operations, which are performed during [8:00,18:00], [8:00,22:00], and [8:00,18:00], respectively. The remaining actions are performed by the Personnel or by the Regulator in the interval [0:00,24:00]. 
Note that the nature of the repair activities and their mutual sequencing constraints are independent of the specific network topology and failed pipe. Moreover, the procedure structure equally fits maintenance interventions for the failures of other network components, such as junctions and valves (Tinga 2012). In fact, whatever the failed item, the network topology must be modified to allow repair, and the supply pressure can be meanwhile increased so as to limit the lack of service, thereby requiring one to perform the inverse operations of topology and pressure restoration.

If the network included multiple supply nodes, a pressure increase should be determined for each of them to guarantee that the minimum pressure constraint is not exceeded at any online load node. The derivation of a pressure increase for each supply node comprises an open problem, totally confined to the fluid-dynamic perspective and beyond the scope of this article. Once a pressure increase $\Delta P_{i}$ is determined for each supply node $S_{i} \in\left\{S_{1}, S_{2}, \ldots, S_{M}\right\}$, a possible solution to accommodate multiple supply nodes in the proposed approach would be to discretize pressure regulation at each supply node into the same number $Z$ of steps, letting step $r \in\{1,2, \ldots, Z\}$ correspond to an increase of $(r / Z) \cdot \Delta P_{i}$ at supply node $S_{i} \forall i \in\{1,2, \ldots, M\}$.

2.2.6 Parametrics. Though avoiding formalization of constraints through a Parametric Diagram, we illustrate here how execution times of maintenance actions are fitted with a different Probability Density Function (PDF) depending on the available statistics. Given that the solution method accepts durations with general (i.e., non-Markovian) distribution, the approach can be easily tailored to any other specific statistics and approximant. Specifically, maintenance actions with deterministic execution time $d$ are associated with a PDF $f(x)=\delta(x-d)$ equal to the Dirac delta function centered at $d$. According to this, all Regulation Steps in Figure 4 have a PDF equal to $\delta(x-2)$. Similarly, Undo Pressure Regulation, which has a deterministic execution time $d$ - stepNumber, depending on the number of completed regulation steps represented by the object node stepNumber, has a PDF equal to $\delta(x-2), \delta(x-4), \delta(x-6)$, and $\delta(x-8)$ depending on whether the value of the integer variable represented stepNumber is $1,2,3$, and 4 , respectively.

When the execution time ranges over a non-pointlike interval without any probabilistic characterization, a uniform distribution is used, as typically done in the literature of stochastic models, often advocating a principle of maximum entropy (Bernardi et al. 2011). This is the case for Pipe Sectioning and Undo Pipe Sectioning, which are associated with a uniform distribution over $[0,1] \mathrm{h}$.

When the execution time ranges over an interval $[a, b]$ and is lower than $c \in(a, b)$ with probability $p$, it can be modeled by a PDF that preserves the finite support $[a, b]$ with null values at the borders and integral over $[a, c]$ equal to $p$, e.g., the expolynomial PDF $f(x)=\alpha(x-a)(b-x) e^{-\lambda x}$ with $\alpha, \lambda \in \mathbb{R}_{0}^{+}$such that $\int_{a}^{b} f(x) d x=1$ and $\int_{a}^{c} f(x) d x=p$. To this end, the system of the latter two equations was solved through Wolfram Mathematica (Wolfram Mathematica 2017) for Preliminary Operations, Pipe Repair, and Concluding Operations, yielding $f(x)=2.4490(x-$ 1) $(3-x) e^{-0.6102 x}, f(x)=0.0347(x-1)(9-x) e^{-0.2245 x}$, and $f(x)=0.8915(x-1)(6-x) e^{-0.9998 x}$, respectively.

\subsection{Formal Stochastic Model}

We translate the composition of the Activity Diagram and the State Charts defined in Section 2.2 into a dynamic Stochastic Time Petri Net (dynamic-STPN) (Carnevali et al. 2018).

2.3.1 Dynamic-STPNs. Syntax. A dynamic-STPN is a tuple $\left\langle P, T, A^{-}, A^{+}, m_{0}, \mathcal{F}, C, E, I, R\right\rangle$, where $P$ and $T$ are the disjoint sets of places and transitions, respectively; $A^{-} \subseteq P \times T$ and $A^{+} \subseteq T \times P$ are the sets of precondition and postcondition arcs, respectively; $m_{0}: P \rightarrow \mathbb{N}$ is the initial marking assigning a number of tokens to each place; $\mathcal{F}: T \rightarrow B^{D}$, with 
$B=[0,1]^{\left[E F T_{t, m}, L F T_{t, m}\right]}$ and $D=\mathbb{N}^{P}$, associates each transition $t$ with a marking-dependent CDF $\mathcal{F}(t): \mathbb{N}^{P} \rightarrow[0,1]^{\left[E F T_{t, m}, L F T_{t, m}\right]}$ which, in turn, associates each marking $m: P \rightarrow \mathbb{N}$ with a CDF $F_{t, m}:\left[E F T_{t, m}, L F T_{t, m}\right] \rightarrow[0,1]$, where $E F T_{t, m} \in \mathbb{Q}_{0}^{+}$and $L F T_{t, m} \in \mathbb{Q}_{0}^{+} \cup\{\infty\}$ are said to be the earliest firing time and the latest firing time of $t$, respectively; $C: T \rightarrow \mathbb{R}^{+}$associates each transition with a weight; $E: T \rightarrow\{\text { true, false }\}^{\mathbb{N}^{P}}$ associates each transition $t$ with an enabling function $E(t): \mathbb{N}^{P} \rightarrow\{$ true, false $\}$ which, in turn, associates each marking with a Boolean value; $I: T \rightarrow \mathbb{R}^{+\mathbb{N}^{P} \times T}$ associates each transition $t$ with a fickle function $I(t):\left(\mathbb{N}^{P} \times T\right) \rightarrow \mathbb{R}^{+}$which, in turn, associates each marking and each (firing) transition with a delay; and $R: T \rightarrow \mathbb{N}$ associates each transition with a priority.

A place $p$ is an input or an output place for a transition $t$ if $\langle p, t\rangle \in A^{-}$or $\langle t, p\rangle \in A^{+}$, respectively. A transition $t$ newly enabled in marking $m$ is termed immediate (IMM) if $\left[E F T_{t, m}, L F T_{t, m}\right]=[0,0]$ and timed otherwise; a timed transition $t$ is EXP if $F_{t, m}(x)=1-e^{-\lambda x}$ over $[0, \infty]$ with $\lambda \in \mathbb{R}_{>0}$ and GEN otherwise; a GEN transition $t$ is DET if $E F T_{t, m}=L F T_{t, m}>0$ and distributed otherwise. If a transition $t$ newly enabled in marking $m$ is distributed, we assume that $F_{t, m}$ is absolutely continuous over its support and thus that there exists a PDF $f_{t, m}$ such that $F_{t, m}(x)=\int_{0}^{x} f_{t, m}(y) d y$.

Semantics. The state of a dynamic-STPN is a pair $\langle m, \tau\rangle$, where $m$ is a marking and $\tau: T \rightarrow \mathbb{R}_{0}^{+}$ associates each enabled transition with a time-to-fire. A transition $t$ is enabled in a state if each of its input places contains at least one token and its enabling function evaluates to true. An enabled transition $t$ is firable if its time-to-fire is equal to 0 and, if $t$ is IMM or DET, if its priority is not smaller than that of any other enabled IMM transition and of any other enabled DET transition with zero time-to-fire. If multiple transitions are firable in $s=\langle m, \tau\rangle$, one of them is selected as the next transition to fire with probability $p_{t}=C(t) / \sum_{t_{i} \in T^{\mathrm{f}}(s)} C\left(t_{i}\right)$, where $T^{\mathrm{f}}(s)$ is the set of firable transitions in $s$. When a transition $t$ fires in a state $s=\langle m, \tau\rangle, s$ is replaced by $s^{\prime}=\left\langle m^{\prime}, \tau^{\prime}\right\rangle$, where

$-m^{\prime}$ is derived from $m$ by removing a token from each input place of $t$, which yields an intermediate marking $m_{\mathrm{tmp}}$, and by adding a token to each output place of $t$;

$-\tau^{\prime}$ is derived from $\tau$ by (i) reducing the time-to-fire of each persistent transition $t_{p}$ (i.e., enabled by $m_{\mathrm{tmp}}$ and $m^{\prime}$ ) by the time elapsed in $s$ (i.e., the time-to-fire of the fired transition $t$ ) and increasing it by the constant value $I\left(t_{p}\right)\left(m^{\prime}, t\right)$; (ii) sampling the time-to-fire of each newly enabled transition $t_{n}$ (i.e., enabled by $m^{\prime}$ but not by $m_{\mathrm{tmp}}$ ) according to $F_{t_{n}, m^{\prime}}$; and (iii) removing the time-to-fire of each disabled transition (i.e., not enabled by $m^{\prime}$ ).

Remark. The features of dynamic-STPNs allow effective modeling of the maintenance procedure: (i) GEN transitions allow representation of repair actions with firmly bounded duration; (ii) enabling functions and fickle functions permit representation of suspension and resumption of repair actions at deterministic time points according to working/overtime hours of personnel; (iii) marking-dependent CDFs allow representation of a different duration of pressure restoration depending on the number of completed regulation steps. Note that, if fickle functions are disregarded and newly enabled transitions are associated with a unique CDF that does not depend on the current marking, dynamic-STPNs reduce to STPNs (Vicario et al. 2009). Also note that dynamicSTPNs presented in Carnevali et al. (2018) include clocks variables, which are omitted here given that they are not used in the procedure modeling.

2.3.2 Stochastic Model Derivation. Figure 5 shows the dynamic-STPN of the procedure specified in Section 2.2, which composes two submodels: the personnel submodel and the activities submodel.

The Personnel Submodel. It is derived from the State Chart of Figure 3(b) and consists of a sequence of DET transitions chained through their input places. Specifically, it includes a 

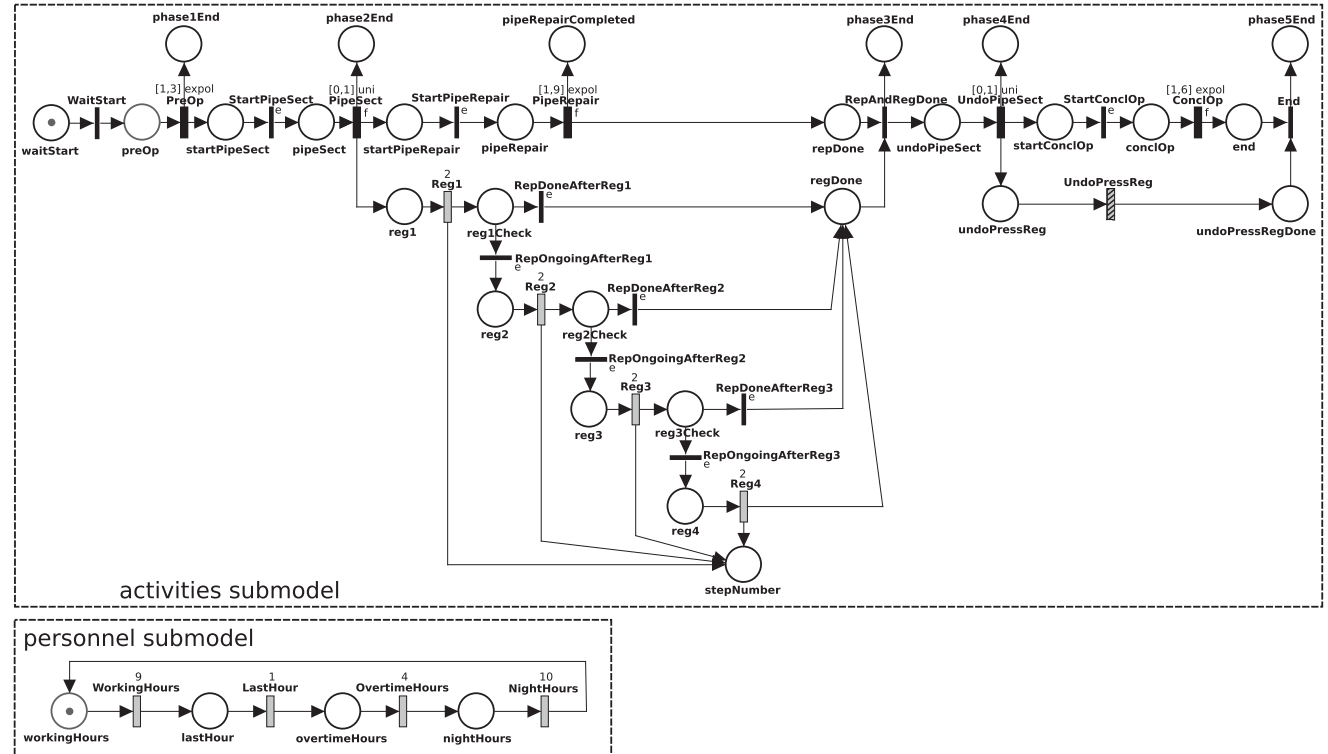

Fig. 5. The dynamic-STPN model of the maintenance procedure specified in Section 2.2. Thin black bars, thick gray bars, and thick black bars represent IMM transitions (i.e., with zero firing time), DET transitions (i.e., with non-zero deterministic firing time), and GEN transitions (i.e., with non-exponentially distributed firing time), respectively; the bar of a transition $t$ has oblique stripes if $t$ has a marking-dependent CDF, and it is marked by a label " $\mathrm{e}$ " or " $\mathrm{f}$ " if $t$ has an enabling or fickle function, respectively.

place for each state in Figure 3(b) modeling working/overtime/night hours and a transition for each transition between these states. According to this, in Figure 5, workingHours, lastHour, overtimeHours, and nightHours are input places for transitions WorkingHours, LastHour, OvertimeHours, and NightHours, respectively, representing the stroke of 17:00, 18:00, 22:00, and 8:00, respectively.

If the procedure starts at 8:00,17:00, 18:00, or 22:00, the initial marking assigns a token to place workingHours, lastHour, overtimeHours, and nightHours, respectively. Otherwise, an additional transition StartHour is chained after transition NightHours, LastHour, or OvertimeHours depending on whether the start time is in (8:00,17:00), (18:00,22:00), and (22:00,8:00), respectively, and the initial marking assigns a token to the output place of StartHour.

The Activities Submodel. It is derived from the Activity Diagram of Figure 4 and the State Charts of Figure 3. For each repair action, it includes a transition with the PDF derived in Section 2.2.6, e.g., transition Pre0p modeling Preliminary Operations has PDF $f(x)=2.4490(x-$ 1) $(3-x) e^{-0.6102 x}$ with $x \in[1,3]$ h. For each structured action, consisting of two sequential actions executed only when the Personnel state represents working/overtime hours, the transition modeling the first action has an enabling function that evaluates to true if the sum of tokens in the corresponding places of the personnel submodel is equal to 1, e.g., transition Conclopstart has an enabling function that evaluates to true if the sum of tokens in places workingHours and lastHour is 1 . Whereas, the transition modeling the second action has a fickle function that shifts its time-to-fire by the duration of the specified non-working hours upon the firing of the transition in the personnel submodel representing the end of the specified working hours, e.g., Conclop has an enabling function that shifts its time to fire by 14 when transition LastHour fires. 
Sequential actions are modeled chaining the corresponding transitions through their input places, e.g., the sequence of Preliminary Operations and Start Pipe Sectioning is modeled making the output place of transition Pre0p be the input place of transition StartPipeSect. A fork node results in a postcondition arc from the transition modeling the input action to the input place of the transitions modeling the output actions, e.g., the fork node with Pipe Sectioning as input and Pipe Repair and Pressure Regulation as output is modeled by the postcondition arc from transition PipeSect to places startPipeRepair and reg1. A join node is accounted for by an IMM transition chaining the output places of the transitions modeling the input actions with the input place of the transition modeling the output action, e.g., the join node with Pipe Repair and Pressure Regulation as input and Undo Pipe Sectioning as output is modeled by transition RepAndRegDone.

Each object node is translated into an absorbing place, e.g., places PipeRepairCompleted and stepNumber. A decision node where the control flows depend on the value of an object node is translated into a switch between IMM transitions having an enabling function whose value depends on the marking of the absorbing place representing the object node, e.g., the decision node between Regulation Steps 1 and 2 is represented by transitions Reg1RepCompleted and Reg1Rep0ngoing, which have an enabling function that evaluates to true if PipeRepairCompleted contains a token and to false otherwise. Given that Undo Pressure Regulation has a different PDF depending on the value of the object node stepNumber, transition undoPressReg has a markingdependent CDF with PDF equal to $\delta(x-2), \delta(x-4), \delta(x-6)$, and $\delta(x-8)$ depending on whether the number of tokens in place stepNumber is equal to $1,2,3$, and 4 , respectively.

According to the State Charts of Figure 3, in the initial state of the system, the clock has just struck 8:00 and the Personnel working hours have just started. Therefore, a DET transition WaitStart is chained with place pre0p in order to model the time to start the maintenance procedure. In the case that the procedure starts at 8:00, WaitStart is an IMM transition (as shown in Figure 5). The initial marking assigns a token to the input place of WaitStart. Note that this additional transition does not increase the complexity of the subsequent stochastic analysis, being just a DET/IMM transition elapsing concurrently with DET transitions of the personnel submodel.

\section{SOLUTION METHOD}

We provide an overview of the overall approach (Section 3.1) and we illustrate how fluid-dynamic analysis of the network (Section 3.2) and stochastic analysis of the procedure (Section 3.3) are combined to evaluate the system performability during maintenance (Section 3.4).

\subsection{A Sketch of the Approach}

The hybrid behavior of a gas distribution network exhibits the continuous dynamics of gas flow and the discrete dynamics of load changes, supply pressure variations, and isolation valve control. In fact, as discussed in Section 2: withdrawn flows at load nodes are continuous variables discretized to reduce the number of configurations for which fluid-dynamic analysis has to be performed; the supply pressure is a continuous variable discretized not only to limit the number of runs of fluid-dynamic analysis but also to determine the duration of Pressure Restoration; and, Pipe Sectioning and Undo Pipe Sectioning are regarded as atomic operations disconnecting the failed pipe from the network and reconnecting it after repair, respectively. While load variations can be assumed to occur regularly at deterministic instants independent of the execution of the maintenance procedure (e.g., every half an hour), valve and pressure control is performed at random times depending on the stochastic duration of repair actions, thus making a gas distribution network subject to maintenance a Stochastic Hybrid System (SHS) (Lygeros and Prandini 2010).

The approach leverages the type of interaction between the continuous and the discrete dynamics of the network to compute performability measures conditioned to a pipe failure (Lopez-Benitez 


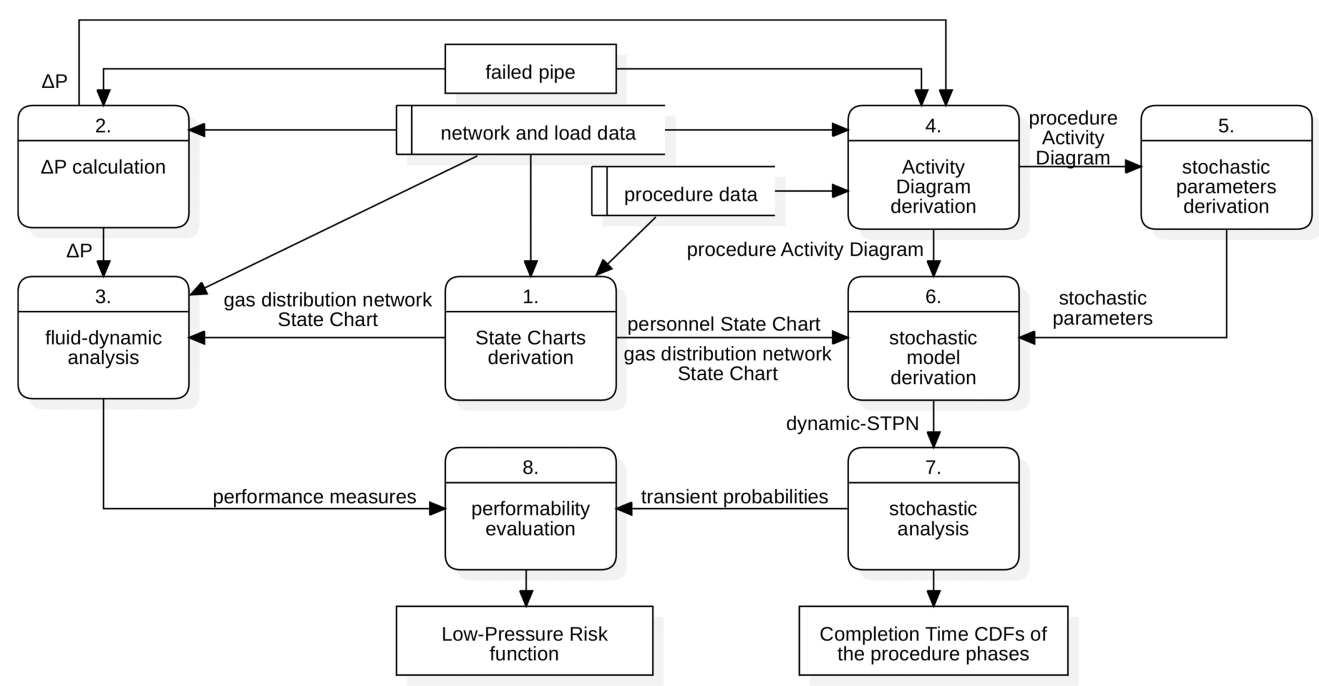

Fig. 6. A dataflow diagram of the proposed method.

and Trivedi 1993). Specifically, after any discrete event, the fluid-dynamic process instantaneously reaches a steady state depending only on the network topology, the supplied pressure, and the gas consumption at that time, neither holding memory of past history nor conditioning the timing of subsequent discrete jumps. Therefore, fluid-dynamic analysis of the gas behavior can be conveniently decoupled from stochastic analysis of the repair procedure, making stochastic analysis almost insensitive to the network size and topology, and thus enabling evaluation of the Low Pressure Risk and the Completion Time CDF of the procedure phases for real scale cases.

Figure 6 illustrates the Dataflow Diagram of the overall methodology:

- On the one hand, fluid-dynamic analysis of the gas distribution network (process 3, Section 3.2) is repeated for each fluid-dynamic configuration determined by the gas distribution network State Chart and composed of (i) the network topology (i.e., regular or modified after pipe sectioning), (ii) the supply pressure (i.e., regular or increased after a regulation step), and (iii) the demand of each load node (i.e., discretized load levels). The results permit computation of performance measures quantifying the risk that pressure goes below a minimum allowed threshold in each configuration that can be reached after a pipe failure.

- On the other hand, stochastic analysis of the maintenance procedure (process 7, Section 3.3) computes transient probabilities of fluid-dynamic configurations, also providing the CDF of the Completion Time of each procedure phase.

-Finally, performability evaluation (process 8, Section 3.4) combines the results of fluiddynamic and stochastic analyses, evaluating the Low-Pressure Risk over time.

In turn, stochastic analysis relies on a dynamic-STPN model of the repair procedure derived from the personnel State Chart, the gas distribution network State Chart, the procedure Activity Diagram, and the stochastic parameters (process 6, Section 2.3). The procedure Activity Diagram is obtained from the procedure data, the network and load data, the identity of the failed pipe, and the pressure increase $\Delta P$ (process 4 , Section 2.2.5), while the stochastic parameters are obtained from the execution times specified in the procedure Activity Diagram (process 5, Section 2.2.6). In turn, the pressure increase $\Delta P$ is derived by iteratively performing fluid-dynamic analysis in the modified network topology for increasing values of the supply pressure until all online load nodes receive 
sufficient pressure (process 2, Section 3.2). Finally, State Charts are derived from the network and load data and the procedure data (process 1, Section 2.2.4). For the sake of space, we avoid including in the Dataflow Diagram the derivation of the Block Diagram, Requirements, and Use Case Diagram.

Note that, beyond the specific application context of gas distribution networks, the solution method can more generally be applied for the performability evaluation of any SHS where (i) discrete transitions occur at deterministic or stochastic times independent of the continuous dynamics and (ii) the continuous behavior between consecutive discrete events is deterministic, independent of the previous continuous state, and univocally determined by the values instantaneously assumed by the continuous variables after the last discrete jump.

\subsection{Fluid-Dynamic Analysis}

Fluid-dynamic analysis derives the pressure $P_{i}$ at each load node $i$ and the flow $Q_{j}$ in each pipe $j$ from a fluid-dynamic configuration made of the network topology, the supply pressure, and the withdrawn flow $Q_{i}^{\mathrm{w}}$ at each load node $i$, the latter being a determined value given that load changes are assumed to occur at deterministic times (e.g., every half an hour). In gas networks working at low pressure, the storage capacity of constituting pipes-sometimes referred to as linepack-is negligible, due to the low difference in density determined by the working conditions. For this reason, the network is considered to react instantly to pressure variations, and fluid-dynamic analysis follows a quasi-static approach. Thus, for each timestep the following equations hold.

- Mass balance at nodes. At each node, the algebraic sum of incoming fluxes must equal the withdrawn flow rate:

$$
\sum_{\mathcal{E}^{+}(i)} Q_{j}-\sum_{\mathcal{E}^{-}(i)} Q_{j}=Q_{i}^{w}
$$

where $Q_{j}$ is the flow rate in pipe $j, \mathcal{E}^{+}(i)$ and $\mathcal{E}^{-}(i)$ are the sets of pipes entering and leaving node $i$, respectively, and $Q_{i}^{w}$ is the flow rate withdrawn at node $i$, which in turn equals the load (if $i$ is a load node) or zero (if $i$ is a junction node).

- Pressure loss in pipes. Pressure varies along each pipe depending on pipe material, diameter, and flow rate, so that pressure difference between start and end nodes is related to flux. The Darcy-Weisbach equation accounts for such dependency in incompressible flow and can be used since the low gas velocity makes compressibility effects negligible:

$$
\Delta P_{i j}=P_{i}-P_{j}=f \frac{L_{i j}}{D_{i j}} \frac{\rho v^{2}}{2},
$$

where $\Delta P_{i j}$ is the pressure difference between node $i$ and node $j, f$ is the Darcy friction factor, $L_{i j}$ and $D_{i j}$ are length and diameter of the pipe connecting nodes $i$ and $j$, respectively, while $\rho$ and $v$ are gas density and velocity, respectively. In turn, the Darcy friction factor $f$ depends on flow characteristic and is calculated through the widely used Colebrook equation (Colebrook 1939), where $\epsilon$ is pipe internal surface roughness and Re is the flow Reynolds number, which relates viscous to inertial forces in the flow:

$$
\frac{1}{\sqrt{f}}=-2 \log \left(\frac{\epsilon / D}{3.71}+\frac{2.51}{\operatorname{Re} \sqrt{f}}\right) .
$$

Equations (1)-(3) are combined in a system where the unknowns are the $N$ node pressures and each node provides one equation (mass balance for junctions and load nodes, and given pressure for supply nodes), yielding a system of $N$ equations. Due to the non-linearity of the problemvelocity is squared in the Darcy-Weisbach equation and the friction factor depends in a non-linear 
fashion on velocity-the solution of each timestep is performed through an iterative procedure based on the Newton-Raphson solution method. This solution technique involves repeatedly calculating Jacobians of a sparse matrix, and each iteration implies solving a linear system in time $O\left(N^{3}\right)$.

The method is implemented in a C\# tool named GasTONE (GAS Tool for the Operation of NEtworks) developed in collaboration with Terranova Software (www. terranovasoftware.eu/eu). The validity of results computed by the tool has been assessed by checking a posteriori that each imposed constraint is satisfied and, for simple topologies, also by manually verifying the analytical solution of the underlying equations. In addition, results have been compared against those obtained through the GeoTech tool, owned by Terranova Software and used by some major gas distribution companies, which is developed independently in a different language $(\mathrm{C}++)$, and proven in use. Results show that the attained accuracy is largely sufficient for the context of use: in particular, for the network considered in Section 4, the worst and average accuracy of the mass balances at nodes is in the order of 0.005 and $3 \times 10^{-5} \mathrm{Nmc} / \mathrm{h}$, respectively, while the difference in terms of pressures at nodes and flow rates in pipes amounts to $0.29 \pm 0.21 \mathrm{mbar}$ and $1.3 \pm 3.3 \mathrm{Nmc} / \mathrm{h}$, respectively.

Moreover, fluid-dynamic analysis is also performed to evaluate the pressure increase $\triangle P$ needed in the supply station during Pressure Regulation in order to restore full service to all users connected to the network during the repair operation. Specifically, fluid-dynamic analysis is performed every time the load changes (e.g., every half an hour) with regular operating pressure by excluding the failed pipe, identifying the most critical time of day in terms of Low-Pressure Risk; then, fluid-dynamic analysis is repeatedly executed, each time increasing the supply pressure until all online nodes have a pressure sufficiently larger than the safety threshold at the most critical time of day. Given that multiple solutions are valid in this case (possibly an infinity of them), more refined optimization methods using fluid-dynamic analysis could be envisaged, so as to minimize some goal variable such as the average network pressure or the global gas leakage.

\subsection{Stochastic Analysis}

Transient analysis of the dynamic-STPN derived in Section 2.3 demands for a solution technique that handles multiple timers with non-Markovian distribution over a bounded support. In principle, approaches based on the approximation of GEN distributions through continuous phasetypes (Horvath et al. 2000; Longo and Scarpa 2009) or solution techniques allowing at most one non-EXP timer enabled in each state (Baier et al. 2017; Lindemann and Thümmler 1999) could be applied for this purpose. However, they would be affected by the presence of concurrent repair actions with firmly bounded execution time and with suspension intervals of deterministic duration scheduled at deterministic time points. Stochastic simulation would avoid approximation of non-EXP distributions, though suffering the need to perform the evaluation for a time span of a few days.

To overcome these issues, evaluation is performed by forward transient analysis based on the method of stochastic state classes (Horváth et al. 2012), which supports quantitative evaluation of models where multiple non-EXP timers can be concurrently enabled in each state. The approach enumerates stochastic state classes that can be reached within a time limit $t_{\max }$, each class encoding the current marking plus the joint support and the joint PDF of the times-to-fire of the enabled transitions and an additional timer measuring the elapsed time. Marking probabilities at $t \in\left[0, t_{\max }\right]$ are computed from the probability that each class is the last node reached within $t$. The approach is implemented in the ORIS Tool (Biagi et al. 2017a; ORIS Tool 2018), which has been proven in use and successfully applied in a variety of applications (Biagi et al. 2017b; Carnevali et al. 2015, 2018). 
Transient probabilities of markings permit derivation of transient probabilities of fluid-dynamic configurations, since each marking corresponds to a set of configurations sharing the same network topology and supply pressure. In fact, if the sum of tokens in places phase1End,..., phase $5 E$ nd is in $\{1,4,5\}$ or in $\{2,3\}$, the network topology is regular or modified, respectively. Moreover, if place phase5End is empty and the number of tokens in place stepNumber is $n \in$ $\{1,2,3,4\}$, the supply pressure is increased by $n \Delta P$, otherwise it is regular. Therefore, the transient probability $p_{\mathrm{y}}(t)$ of each fluid-dynamic configuration $\mathrm{y}$ can be derived from the transient probability of the set $\mathcal{M}_{\mathrm{y}}$ of markings corresponding to the same network topology and supply pressure of $y$ :

$$
p_{\mathbf{y}}(t)=I(\mathrm{y}, t) \cdot \sum_{m \in \mathcal{M}_{\mathrm{y}}} p_{m}(t),
$$

where $I(\mathrm{y}, t)$ is an indicator function that evaluates to 1 if the withdrawn flows at load nodes at time $t$ are those of configuration $\mathbf{y}$, and evaluates to zero otherwise.

CDFs of the completion times of the procedure phases are also an outcome of stochastic analysis, and can be useful for network operators to support decisions and in the interaction with other subjects. In particular, the CDF of the completion time of the $N$-th phase can be evaluated as the transient probability of any marking such that phase NEnd contains a token, $\forall N \in\{1,2,3,4,5\}$.

\subsection{Performability Evaluation}

We characterize the network performability in the transient phase following a pipe failure by evaluating the risk that users are served with insufficient pressure. To this end, we define a LowPressure Risk (LPR) function that takes into account, for each load node at each time, how far the current pressure is from a minimum value required to guarantee device operation and safety, and from a maximum value needed to supply correct service:

$$
\operatorname{LPR}(t)=\sum_{i \mid P_{i}(t)<P_{\mathrm{thr}}} \frac{\left(P_{\mathrm{thr}}-P_{i}(t)\right)^{\alpha}}{P_{i}(t)-P_{\min }},
$$

where $P_{i}(t)$ is the pressure at load node $i$ at time $t$ (computed by fluid-dynamic analysis), $P_{\min }$ is the minimum pressure never to be attained for safe operation, $P_{\text {thr }}$ is the pressure above which the risk is considered null, and $\alpha$ is an exponent that can be adjusted to change the shape of the risk function, which has an asymptote at $P_{i}(t)=P_{\min }$, and null value and derivative at $P_{i}(t)=P_{\mathrm{thr}}$. In particular, the larger $\alpha$, the larger the weight of nodes with pressure closer to the minimum. For any time $t$, we evaluate the expected value of $\operatorname{LPR}(t)$, which we denote as $\Gamma(t)$ :

$$
\Gamma(t) \stackrel{\text { def }}{=} \mathrm{E}[\operatorname{LPR}(t)]=\int_{\mathbb{R}_{0}^{+}} f_{\mathrm{LPR}(t)}(x) d x
$$

where $f_{\mathrm{LPR}(t)}$ is the $\mathrm{PDF}$ of $\operatorname{LPR}(t)$. According to the law of total probability,

$$
\Gamma(t)=\int_{\mathbb{R}_{0}^{+}} x \int_{\Gamma} f_{\mathrm{LPR}(t) \mid \mathrm{y}}(x) f_{\gamma(t)}(\mathbf{y}) d \mathbf{y} d x,
$$

where $\gamma(t) \in \Gamma$ is a fluid-dynamic configuration made of a Boolean value $\varphi(t)$ indicating whether the network topology at time $t$ is regular or modified due to pipe sectioning; a continuous variable $\rho(t)$ representing the supply pressure at time $t$, either regular or increased after a regulation step; and, the withdrawn flow $Q_{i}^{\mathrm{w}}(t)$ at each load node $i$ at time $t$, which, as already mentioned, is a determined value given that load changes are assumed to occur at deterministic times (e.g., every 
half an hour). Note that

$$
\Gamma(t)=\int_{\Gamma} f_{\gamma(t)}(\mathbf{y}) \int_{\mathbb{R}_{0}^{+}} x f_{\mathrm{LPR}(t) \mid \mathbf{y}}(x) d x d \mathbf{y},
$$

where $\operatorname{LPR}(t) \mid \mathbf{y}=\sum_{i \mid\left(P_{i}(t) \mid \mathbf{y}\right)<\eta} \omega\left(P_{i}(t) \mid \mathbf{y}\right)$ is a determined value because $P_{i}(t)$ is univocally derived by fluid-dynamic analysis from the configuration $\mathrm{y}$ at time $t$. According to this, the PDF of $\operatorname{LPR}(t) \mid \mathrm{y}$ is $f_{\mathrm{LPR}(t) \mid \mathrm{y}}(x)=\delta(x-(\operatorname{LPR}(t) \mid \mathrm{y}))$, and we obtain

$$
\Gamma(t)=\int_{\Gamma} f_{\gamma(t)}(\mathrm{y}) \operatorname{LPR}(t) \mid \mathbf{y} d \mathbf{y} .
$$

Also note that $\operatorname{LPR}(t) \mid \mathrm{y}$ takes the same value $\forall t \mid \gamma(t)=\mathrm{y}$, due to the fact that the fluid-dynamic process immediately reaches a steady state after any discrete event changes the fluid-dynamic configuration. Therefore, the expression can be rewritten as

$$
\Gamma(t)=\int_{\Gamma} f_{\gamma(t)}(\mathrm{y}) \operatorname{LPR} \mid \mathbf{y} d \mathbf{y},
$$

where the integral is computed by discretization of time. On the one hand, fluid-dynamic analysis is repeated for each fluid-dynamic configuration $\mathrm{y} \in \Gamma$ to derive performability measures LPR $\mid \mathrm{y}$. On the other hand, stochastic analysis computes $f_{\gamma(t)}$ at equidistant time points within the time horizon of interest, deriving the transient probability $p_{\mathrm{y}}(t)$ of each fluid-dynamic configuration y. In so doing, $\Gamma(t)$ can be derived by weighting the performability measures according to their respective transient probability (process 7 in Figure 6):

$$
\Gamma(t)=\sum_{\mathbf{y} \in \Gamma} p_{\mathbf{y}}(t) \operatorname{LPR} \mid \mathbf{y} .
$$

Overall, the Low-Pressure Risk LPR $(t)$ and the CDF of the completion time of each repair phase can be used to compare different maintenance and scheduling strategies, supporting decisions in the organization of repair procedures. It should be remarked that, in principle, solutions that are optimal with respect to duration (e.g., low impact on traffic thanks to limited road rerouting) might not be optimal with regard to customer impact and vice versa, leading to tradeoff solutions.

\section{CASE STUDY}

We report results of application on a case study aimed at illustrating the solution method and the performability measures it permits to compute, providing guidance on the selection of the model parameters and demonstrating feasibility of the approach on a case of real scale. To this end, we consider a typical multi-phased maintenance procedure for a real gas distribution network located in Poland, using actual load profiles for domestic, office, and industrial users (Section 4.1). The network performability during the procedure is evaluated by computing the expected LPR over time and the CDF of the completion time of the procedure phases, so as to assess the impact on the network users and on people frequenting the area near the failure location, respectively. To support the selection of parameters, a sensitivity analysis is performed (Section 4.2) taking into account the number of steps considered for pressure regulation (i.e., a parameter of both the network model and the procedure model, thus affecting both fluid-dynamic analysis and stochastic analysis), the time granularity of load profiles (i.e., a parameter of the network model only), and the execution time distribution of maintenance actions (i.e., parameters of the procedure model only). Experimental results show that 32 regulation steps, a time granularity of 30min, and expolynomial distributions (or a mixture of uniform distributions) guarantee the accuracy requested in the context of gas distribution networks for the evaluation of performability measures (Section 4.3). Moreover, thanks to the limited computational complexity, performability evaluation 


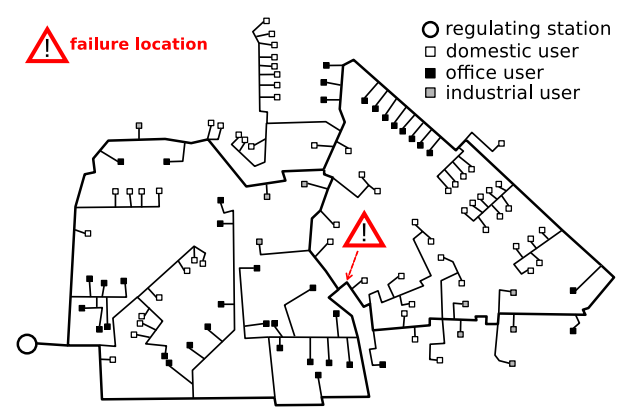

(a)

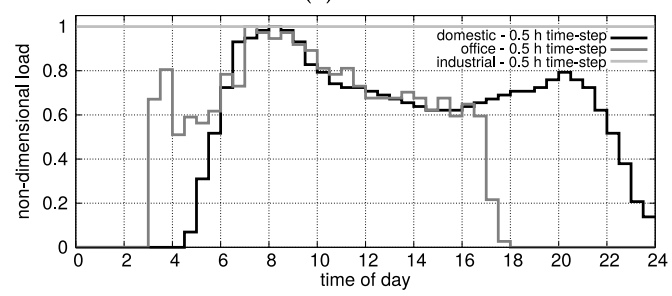

(c)

\begin{tabular}{|l|c|}
\hline Supply nodes & 1 \\
Junction nodes & 207 \\
Load nodes & 108 \\
\hline Residential load nodes & 60 \\
Office load nodes & 39 \\
Constant load nodes & 9 \\
\hline Pipes & 319 \\
Pipes inner diameter range & $51.4-204.6 \mathrm{~mm}$ \\
\hline Minimum required pressure & $17.0 \mathrm{mbar}_{\mathrm{g}}$ \\
\hline Total load (for a winter day at 7:00) & $321.5 \mathrm{Nm}^{3} / \mathrm{h}$ \\
\hline
\end{tabular}

(b)

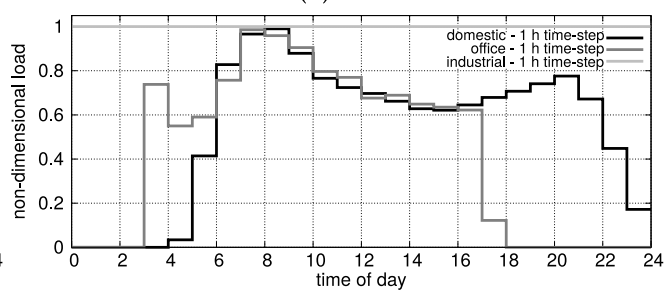

(d)

Fig. 7. (a) Topology of the gas distribution network of Szoplik (2012). (b) Network characteristics: shaded rows refer to data available in Szoplik (2012); non-shaded rows show data selected by preserving information disclosed in Szoplik (2012). (c) Load profiles with $0.5 \mathrm{~h}$ timestep and (d) $1 \mathrm{~h}$ timestep.

can be repeated for different start times of the maintenance procedure in order to select the one that minimizes the risk of violating the minimum pressure constraint. Experimental results show that the start time of the procedure indeed has a significant impact on the quality of service experienced by the network users, thus comprising a relevant parameter in the planning of maintenance operations. Experiments are performed on a machine equipped with an Intel Xeon $2.67 \mathrm{GHz}$ and 32.0GB RAM.

\subsection{Network Model}

Figure 7(a) shows a topological representation of the network of Szoplik (2012), belonging to the gas distribution network of Szczecin, Poland. The available network characteristics are shown in Figure 7(b) (the length and diameter of 26 out of 319 pipes and the total length of the pipes having a specific diameter are not shown due to space reasons). Among missing data, pipe lengths are estimated by properly scaling the network layout, and pipe diameters are assigned considering the available total lengths and keeping the gas velocity in pipes below $2 \mathrm{~m} \mathrm{~s}^{-1}$ to avoid excessive pressure losses and dust issues. In the cases where a graph partition exists such that the flow of all cut edges is available, the maximum load of nodes is estimated computing a mass balance (similar to Equation (1)) on single nodes or on small node clusters, calculating the algebraic sum of flows entering the partition and assigning the corresponding load to the contained nodes. Otherwise, node loads are estimated so as to keep gas velocity in pipes below $\mathrm{m} \mathrm{s}^{-1}$ and to be consistent with the total load.

Nodes are cast into three classes each featuring a different load profile, following a practice commonly adopted by network operators, e.g., to plan network operation or to cope with missing meter reading when billing. Figure $7(\mathrm{c})$ and (d) shows the non-dimensional load of each class for $\Delta t=0.5 \mathrm{~h}$ and $\Delta t=1 \mathrm{~h}$, respectively: the domestic load is based on data of a typical domestic 
user, with a double peak in the early morning and in the evening; the office load is derived from data reported for a government building in Glasgow, Scotland, and features a peak in the early morning and very low consumption in the evening (Government 2014); and, the constant load is chosen as a simplified pattern to represent industrial use. Moreover, parameters for calculating the Low-Pressure Risk according to Equation (5) are $P_{\min }=18.0 \mathrm{mbar}, P_{\mathrm{thr}}=20.0 \mathrm{mbar}$, and $\alpha=1.5$; and, the minimum allowed pressure for the computation of $\Delta P$ is assumed to be equal to $P_{\text {thr }}$.

\subsection{Sensitivity Analysis}

We consider a number of steps $S \in\{2,4,8,16,32\}$ for pressure regulation. Moreover, we consider a timestep $\Delta t \in\{0.5 \mathrm{~h}, 1 \mathrm{~h}, 2 \mathrm{~h}, 4 \mathrm{~h}\}$ for load data: starting from half-hourly data, load for larger timesteps is estimated as the average of the half-hourly values during each timestep, thus assuring that the total consumption is invariant in the four cases, e.g., the hourly load in $[14: 00,15: 00]$ is the average of the half-hourly load in [14:00,14:30] and [14:30,15:00]. Furthermore, for repair actions with an execution time that ranges in a time interval $[a, b]$ and is not greater than $c \in(a, b)$ with probability $p$ (i.e., Preliminary Operations, Pipe Repair, and Concluding Operations), we consider (i) the expolynomial PDF $f(x)=\alpha(x-a)(b-x) e^{-\lambda x}$ with $\alpha, \lambda \in \mathbb{R}_{0}^{+}$ such that $\int_{a}^{c} f(x) d x=p$, presented in Section 2.2.6 (expol-case); (ii) a mixture of uniform PDFs $f(x)=p \cdot u(a, c)+(1-p) u(c, b)$, where $u(\min , \max )$ is the uniform PDF over [min, max] (unifcase); and (iii) a mixture of Dirac delta functions $f(x)=p \cdot \delta(x-c)+(1-p) \delta(x-b)$ (det-case). In the det-case, transitions with deterministic firing time in the activities submodel are associated with larger priority than those in the personnel submodel, to avoid non-determinism and reduce the state-space size.

Note that, if the number of regulation steps varies, then both fluid-dynamic analysis (illustrated in Section 3.2) and stochastic analysis (illustrated in Section 3.3) must be repeated. Conversely, variations in the time granularity of load profiles require only fluid-dynamic analysis to be repeated, whereas variations in the PDF of repair actions require only stochastic analysis to be repeated. Obviously, in all these cases, performability evaluation (illustrated in Section 3.4) must be rerun.

\subsection{Experimental Results}

Stochastic analysis takes nearly $16 \mathrm{~min}$ to evaluate the expol-case model with procedure start at 8:00, time limit $72 \mathrm{~h}$, and computation step $0.1 \mathrm{~h}$ (i.e., the timestep for the numerical computation of transient probabilities). The analysis results yield the transient probability of each fluid-dynamic configuration and the completion time CDF of the procedure phases. For the expol-case, Figure 8(a) shows the completion time $\mathrm{CDF}$ of the phases that change the network topology (i.e., phases 2 and 4 ) and the phase that ends the procedure (i.e., phase 5). While phases 1 and 2 are terminated within day 1, phase 5 is likely to be completed in day 2, making the night-time stop clearly visible. Note that all curves appear continuous in time, given that the completion time CDF of each phase does not depend on discrete-time load variations, but only on continuous-time PDFs of execution times of repair actions and on sequencing constraints among them.

To evaluate the risk that gas is supplied with insufficient pressure during pipe maintenance, we consider the failure of the pipe marked in Figure 7(a), which in fact emerges from a preliminary analysis as one of the most critical failures in terms of low-pressure risk. For the case with 32 regulation steps and 30min timestep, fluid-dynamic analysis is repeated for the 48 half-hours of the day with regular supply pressure by excluding the failed pipe, requiring nearly $12 \mathrm{~s}$. The analysis results yield the most critical time of day and node in terms of pressure, and the pressure increase $\Delta P=2.0 \mathrm{mbar}$. Then, fluid-dynamic analysis of the network in the modified topology is repeated for each fluid-dynamic configuration determined by the 32 pressure levels and the 


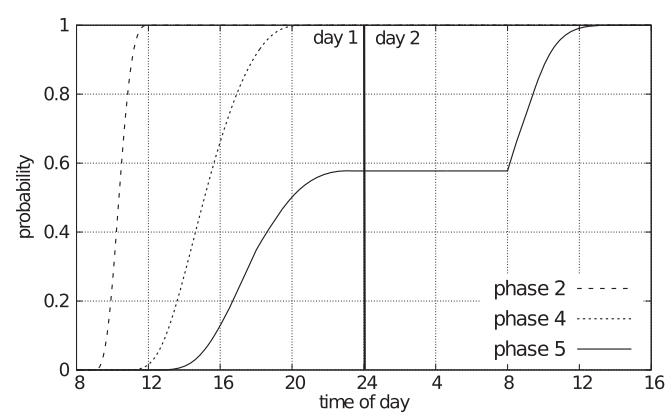

(a)

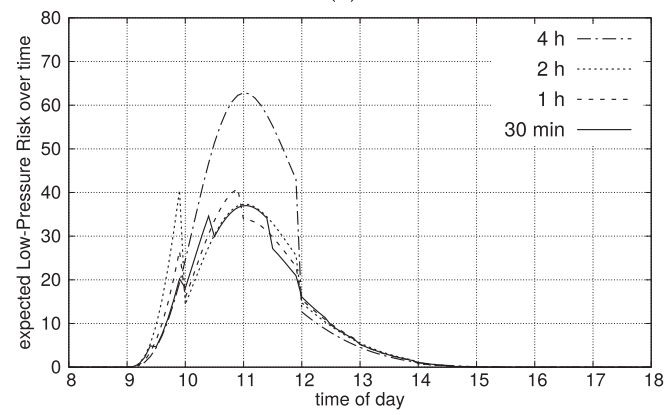

(c)

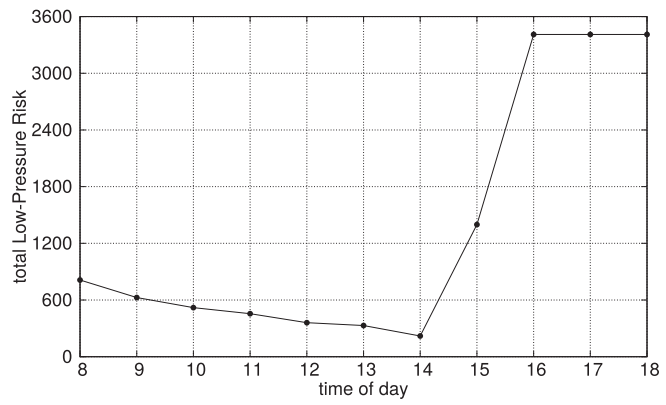

(e)

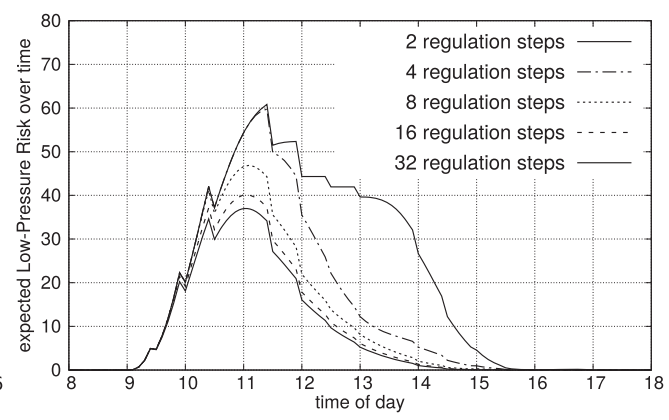

(b)

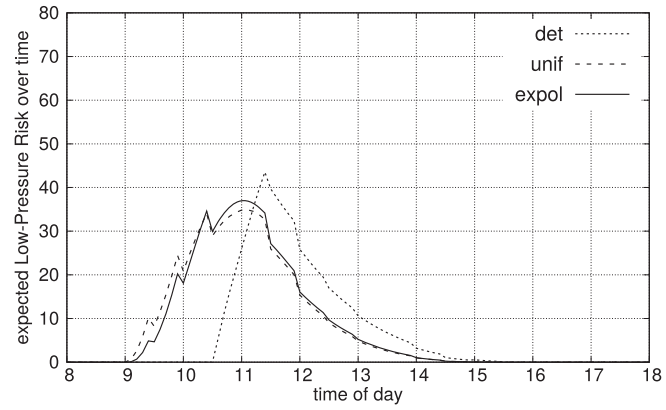

(d)

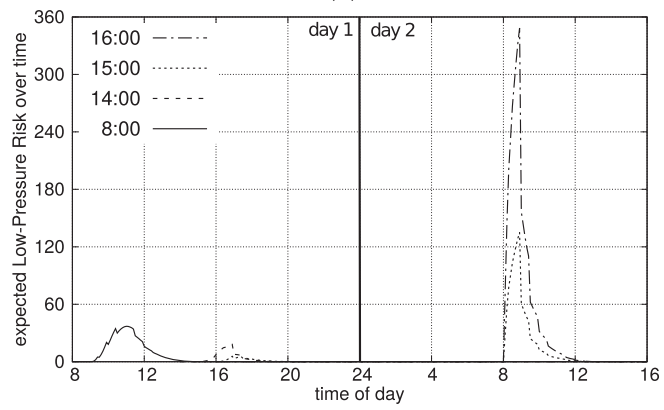

(f)

Fig. 8. (a) Completion time CDF of phases 2, 4, and 5 for the expol-case with procedure start at 8:00 and 32 regulation steps. With procedure start at 8:00, $\Gamma(t)$ computed for (b) the expol-case with 30min timestep for load discretization and varying number of regulation steps; (c) the expol-case with 32 regulation steps and varying timestep; and (d) the expol-, unif-, and det- cases with 32 regulation steps and 30min timestep. (e) The integral of $\Gamma(t)$ for the expol-case with 32 regulation steps, 30min timestep, and varying starting hour. (f) $\Gamma(t)$ for the expol-case with 32 regulation steps, 30min timestep, and varying starting hour.

48 half-hours of the day; the total computation time for the resulting 1,536 runs is about $6 \mathrm{~min}$. For each run, corresponding to a fluid-dynamic configuration $\mathbf{y}$, node pressures are derived, and thus LPR $\mid \mathrm{y}$ is calculated. Using the transient probability $p_{\mathrm{y}}(t)$ of each configuration $\mathbf{y}$ computed by stochastic analysis, $\Gamma(t)$ is finally derived according to Equation (11).

For the expol-case with procedure start at 8:00, Figure 8(b) shows $\Gamma(t)$ computed with $30 \mathrm{~min}$ timestep for load discretization and varying number of regulation steps, while Figure $8(\mathrm{c})$ shows $\Gamma(t)$ computed with 32 regulation steps and varying timestep. Given that $\Gamma(t)$ is determined by transient probabilities of fluid-dynamic configurations, which in turn depend on the time of day 
Table 2. Accuracy of $\Gamma(T)$ Computed for the Expol-Case with 30min Timestep and Increasing Number of Regulation Steps (Columns 2-5), and with 32 Regulation Steps and

Decreasing Timestep (Columns 6-8)

\begin{tabular}{|c|c|c|c|c|c|c|c|}
\cline { 2 - 8 } \multicolumn{1}{c|}{} & \multicolumn{3}{c|}{$\operatorname{RMAD}\left(\Gamma(t)_{n, 30 \min }, \Gamma(t)_{2 n, 30 \min }\right)$} & \multicolumn{3}{c|}{$\operatorname{RMAD}\left(\Gamma(t)_{32, \Delta t}, \Gamma(t)_{32, \Delta t / 2}\right)$} \\
\hline Procedure start time & $n=2$ & $n=4$ & $n=8$ & $n=16$ & $\Delta t=4 \mathrm{~h}$ & $\Delta t=2 \mathrm{~h}$ & $\Delta t=1 \mathrm{~h}$ \\
\hline $8: 00$ & 0.1763 & 0.1565 & 0.0507 & 0.0190 & 0.0488 & 0.0143 & 0.0151 \\
\hline $9: 00$ & 0.1560 & 0.0953 & 0.0507 & 0.0207 & 0.0352 & 0.0162 & 0.0112 \\
\hline $10: 00$ & 0.2267 & 0.1284 & 0.0302 & 0.0131 & 0.0319 & 0.0169 & 0.0068 \\
\hline $11: 00$ & 0.3888 & 0.2023 & 0.0278 & 0.0130 & 0.0271 & 0.0862 & 0.0092 \\
\hline $12: 00$ & 0.5064 & 0.2478 & 0.0448 & 0.0158 & 0.0424 & 0.0613 & 0.0151 \\
\hline $13: 00$ & 0.4481 & 0.3158 & 0.0566 & 0.0201 & 0.0661 & 0.0348 & 0.0113 \\
\hline $14: 00$ & 0.4075 & 0.2650 & 0.0455 & 0.0202 & 0.0566 & 0.0305 & 0.0115 \\
\hline $15: 00$ & 0.6779 & 0.3880 & 0.0927 & 0.0381 & 0.0897 & 0.0490 & 0.0195 \\
\hline $16: 00$ & 0.3586 & 0.1978 & 0.0705 & 0.0233 & 0.0470 & 0.0216 & 0.0092 \\
\hline $17: 00$ & 0.3586 & 0.1978 & 0.0705 & 0.0233 & 0.0470 & 0.0216 & 0.0092 \\
\hline $18: 00$ & 0.3586 & 0.1978 & 0.0705 & 0.0233 & 0.0470 & 0.0216 & 0.0092 \\
\hline
\end{tabular}

at which gas demand changes, all curves show discontinuities (nearly) every half-hour due to the discretized change in load levels. The overall shape is affected by the timing of Preliminary Operations: $\Gamma(t)$ starts rising shortly after 9:00 (Preliminary Operations take at least $1 \mathrm{~h}$ ), indicating an increasing probability of having completed Pipe Sectioning and thus of experiencing some LPR; after 11:00/11:30, measures keep decreasing due to the lower LPR corresponding to the afternoon hours and due to the increasing probability of having completed many regulation steps.

Due to the rough representation of pressure regulation, the curve of the case with two regulation steps in Figure 8(b) significantly differs from the other ones, especially after 11:00/11:30 when the probability of having completed many regulation steps increases. Similarly, the curve with $4 \mathrm{~h}$ timestep in Figure 8(c) suffers the rough load discretization, especially in the range [8:00,12:00] during which the value of $\Gamma(t)$ is larger. With a larger number of regulation steps or a finer load discretization, the expected LPR over time is captured more closely. To quantitatively support selection of the model parameters, the Relative Mean Absolute Deviation (RMAD) is used to measure the difference between time series values of $\Gamma(t)$ computed for the time values of interest $t=t_{1}, \ldots, t_{N}$ with a different number of regulation steps and with a different timestep for load discretization:

$$
\operatorname{RMAD}\left(\Gamma(t)_{n_{i}, \Delta t_{i}}, \Gamma(t)_{n_{j}, \Delta t_{j}}\right)=\frac{1}{N} \sum_{t=t_{1}}^{t_{N}}\left|\frac{\Gamma(t)_{n_{i}, \Delta t_{i}}-\Gamma(t)_{n_{j}, \Delta t_{j}}}{\Gamma(t)_{n_{j}, \Delta t_{j}}}\right|,
$$

where $\Gamma(t)_{n_{i}, \Delta t_{i}}$ is $\Gamma(t)$ computed with $n_{i}$ regulation steps and timestep $\Delta t_{i}$ (not to burden notation, we avoid specifying the distribution of repair actions and the procedure start time). Table 2 shows the RMAD between time series values of $\Gamma(t)$ computed for the expolcase with different procedure start time and with (i) 30min timestep and increasing number of regulation steps (columns 2-5), i.e., $\operatorname{RMAD}\left(\Gamma(t)_{n, 30 \mathrm{~min}}, \Gamma(t)_{2 n, 30 \mathrm{~min}}\right)$ with $n \in\{2,4,8,16\}$, and (ii) 32 regulation steps and decreasing timestep (columns 6-8), i.e., $\operatorname{RMAD}\left(\Gamma(t)_{32, \Delta t}, \Gamma(t)_{32, \Delta t / 2}\right.$ ) with $\Delta t \in\{4,2,1\} \mathrm{h}$. As expected from the curves shown in Figure 8(b) and (c), the RMDA decreases as the number of regulation steps increases and the timestep decreases. In particular, $\operatorname{RMAD}\left(\Gamma(t)_{16,30 \mathrm{~min}}, \Gamma(t)_{32,30 \mathrm{~min}}\right)$ and $\operatorname{RMAD}\left(\Gamma(t)_{32,1 \mathrm{~h}}, \Gamma(t)_{32,30 \mathrm{~min}}\right)$ are below 0.04 for all start times, 
showing that 32 regulation steps and 30min timestep are largely sufficient to attain the accuracy requested by the context of use.

Figure $8(\mathrm{~d})$ shows $\Gamma(t) \mathrm{d}$ for the expol-, unif-, and det- cases with 32 regulation steps, 30min timestep, and procedure start at 8:00. On the one hand, the expol-case and the unif-case provide finer estimation of the expected LPR over time, showing quite close trends, which can be explained by the fact that, for each repair action subject to sensitivity analysis (i.e., repair actions with an execution time ranging over $[a, b]$ and not greater than $c \in(a, b)$ with probability $p)$, both the PDF of the expol-case and the PDF of the unif-case have integrals equal to $p$ and $1-p$ over the contiguous intervals $[a, c]$ and $[c, b]$, respectively. Conversely, the curve of the det-case shows a significant delay in time, since the probability masses $p$ and $1-p$ are not spread over the above-mentioned intervals, but rather concentrated at their upper boundaries $c$ and $b$, respectively. On the other hand, in the det-case, the computation time of stochastic analysis is one order of magnitude lower than in the unif-case and the expol-case, e.g., in the setting of Figure 8(d) stochastic analysis takes nearly $35 \mathrm{~s}, 7 \mathrm{~min}$, and $16 \mathrm{~min}$ in the det-, unif-, and expol-case, respectively. According to this, in the early stages of definition of the maintenance procedure, the rough yet fast evaluation provided by the det-case turns out useful to support iterative refinement of design choices, while accurate evaluation of the final specification can be performed through the expol-case or the unif-case.

Owing to the limited computational complexity, the evaluation can be repeated in order to determine the optimal start time of the procedure. To this end, as a global measure of the procedure impact, we consider the integral of $\Gamma(t)$ over a time interval $\tau$ sufficiently wide to guarantee the procedure completion, i.e., $\gamma=\int_{\tau} \Gamma(t) d t$. Specifically, for the expol-case, Figure 8(e) plots $\gamma$ computed for $\tau=[0,72] \mathrm{h}$ (measured since the procedure start time), 32 regulation steps, 30min timestep, and start time in $\{8: 00,9: 00, \ldots, 18: 00\}$. As the procedure start time increases from 8:00 on, the value of $\gamma$ decreases, due to the lower probability of completing Pipe Sectioning in the time interval [8:00,14:00] during which gas consumption is larger. Starting the procedure from 15:00 on, $\gamma$ significantly increases due to the larger probability of starting Pipe Sectioning after 8:00 of day 2, as also shown by the plots of $\Gamma(t)$ in Figure $8(\mathrm{f})$. In particular, the value of $\gamma$ for start time equal to $16: 00,17: 00$, and 18:00 is the same due to the null probability of starting Pipe Sectioning on day 1.

\section{DISCUSSION}

This work presents a methodology for the evaluation of repair procedures, developed within a research collaboration with an industrial company delivering added-value services for gas distribution operators. Specifically, an accurate formal model derived from a SysML semi-formal specification and an efficient solution technique are integrated into an organic toolchain, with substantial invariance of complexity with respect to the network size and topology, providing insight into the phenomena affecting the computed quantitative measures and guiding network operators on data to capture. The approach evaluates the performance of the procedure, providing the completion time PDF of its phases, and assesses the impact on the quality of service delivered to users, evaluating the risk that gas is supplied with insufficient pressure. The evaluation is repeated for different start times of the procedure to determine the optimal time of day for the maintenance process to start. The obtained results could be used to validate repair procedures, hence supporting planning of ordinary and preventive maintenance, analysis of investment options for network strengthening, and budget allocation to improve the performance of repair activities.

The approach proposed to model maintenance procedures for gas distribution networks can be applied to a wider class of problems in the area of phased mission systems (Wang and Trivedi 2007), where consecutive, non-overlapping operation phases with stochastic duration are performed 
under different working conditions and performability requirements (e.g., the flight of an aircraft consisting of taking off, ascending, cruising, descending, and landing phases). Specifically, SysML artifacts can be used to provide a semi-formal specification of the overall mission, using a Block Diagram to specify the system resources (e.g., engines), State Charts to represent the behavior of such resources in the different operation phases (e.g., engines may have larger failure probability in the taking off phase), and an Activity Diagram to detail each operation and the involved resources (e.g., a single engine may be sufficient for the cruising phase while multiple may be required for taking off and landing). Then, a formal stochastic model can be derived applying the same transformation rules devised here, enabling performability evaluation based on stochastic analysis.

The most relevant improvement for the full exploitation of the proposed methodology is the availability of finer statistics of maintenance durations, which may permit fitting of execution times with more accurate stochastic distributions. Actually, the growing deployment of smart technologies in the gas distribution domain is making these data easier to capture by network operators. The availability of statistical data or prediction of failure probabilities and failure costs incurred could enable a probabilistic approach to Failure Modes and Effects Analysis (FMEA), supporting quantitative assessment of potential risks, sensitivity analysis with respect to network parameters, and long-term evaluation of average measures of service lack and consequent overhead.

\section{REFERENCES}

Gaetano Abbatantuono, Silvio Lamonaca, Massimo La Scala, and Ugo Stecchi. 2016. Monitoring and emergency control of natural gas distribution urban networks. In 2016 IEEE Workshop on Environmental, Energy, and Structural Monitoring Systems (EESMS'16). 1-6. DOI : https://doi.org/10.1109/EESMS.2016.7504843

AEEGSI 2017. Homepage. Retrieved on June 1, 2017 from http://www.autorita.energia.it/it/inglese/about/presentazione. htm.

Christel Baier, Clemens Dubslaff, Lubos Korenciak, Antonín Kucera, and Vojtech Rehák. 2017. Mean-payoff optimization in continuous-time Markov chains with parametric alarms. In International Conference on Quantitative Evaluation of Systems. 190-206. DOI : https://doi.org/10.1007/978-3-319-66335-7_12

Lorenzo Baldacci, Matteo Golfarelli, Davide Lombardi, and Franco Sami. 2016. Natural gas consumption forecasting for anomaly detection. Expert Systems with Applications 62 (2016), 190-201. DOI : https://doi.org/10.1016/j.eswa.2016.06.013

Simonetta Balsamo, Antinisca Di Marco, Paola Inverardi, and Marta Simeoni. 2004. Model-based performance prediction in software development: A survey. IEEE Transactions on Software Engineering 30, 5 (May 2004), 295-310. DOI : https:// doi.org/10.1109/TSE.2004.9

Simona Bernardi, Javier Campos, and José Merseguer. 2011. Timing-failure risk assessment of UML design using time Petri net bound techniques. IEEE Transactions on Industrial Informatics 7, 1 (2011), 90-104. DOI : https://doi.org/10.1109/TII. 2010.2098415

Simona Bernardi, Susanna Donatelli, and José Merseguer. 2002. From UML sequence diagrams and statecharts to analysable Petri net models. In International Workshop on Software and Performance. ACM, 35-45. DOI : https://doi.org/10.1145/ 584369.584376

Simona Bernardi, José Merseguer, and Dorina C. Petriu. 2013. Model-Driven Dependability Assessment of Software Systems. Springer. DOI : https://doi.org/10.1007/978-3-642-39512-3

Marco Biagi, Laura Carnevali, Marco Paolieri, and Enrico Vicario. 2017a. An introdution to the ORIS tool. In EAI International Conference on Performance Evaluation Methodologies and Tools. ACM, 9-11. DOI : https://doi.org/10.1145/3150928. 3158361

Marco Biagi, Laura Carnevali, Marco Paolieri, and Enrico Vicario. 2017b. Performability evaluation of the ERTMS/ETCS - Level 3. Transp.ortation Research Part C: Emerging Technologies 82 (2017), 314-336. DOI : https://doi.org/10.1016/j.trc. 2017.07.002

Marco Biagi, Enrico Vicario, and Reinhard German. 2018. Extending the steady state analysis of hierarchical semi-Markov processes with parallel regions. In European Workshop on Computer Performance Engineering.

Andrea Bobbio, Antonio Puliafito, and Miklós Tekel. 2000. A modeling framework to implement preemption policies in nonMarkovian SPNs. IEEE Transactions on Software Engineering 26, 1 (2000), 36-54. DOI : https://doi.org/10.1109/32.825765

Anderson J. Brito, Adiel Teixeira de Almeida, and Caroline M. M. Mota. 2010. A multicriteria model for risk sorting of natural gas pipelines based on ELECTRE TRI integrating utility theory. European fournal of Operational Research 200, 3 (2010), 812-821. DOI : https://doi.org/10.1016/j.ejor.2009.01.016 
Enrico Cagno, Franco Caron, Mauro Mancini, and Fabrizio Ruggeri. 2000. Using AHP in determining the prior distributions on gas pipeline failures in a robust Bayesian approach. Reliability Engineering \& System Safety 67, 3 (2000), 275-284. DOI : https://doi.org/10.1016/S0951-8320(99)00070-8

Laura Carnevali, Christopher Nugent, Fulvio Patara, and Enrico Vicario. 2015. A continuous-time model-based approach to activity recognition for ambient assisted living. In International Conference on Quantitative Evaluation of Systems. Springer, 38-53. DOI : https://doi.org/10.1007/978-3-319-22264-6_3

Laura Carnevali, Marco Paolieri, Fabio Tarani, Enrico Vicario, and Kumiko Tadano. 2014. Modeling and evaluation of maintenance procedures for gas distribution networks with time-dependent parameters. In Computer Safety, Reliability, and Security, Lecture Notes in Computer Science, Vol. 8696.304-315. DOI : https://doi.org/10.1007/978-3-319-10557-4_34

Laura Carnevali, Fabio Tarani, and Enrico Vicario. 2018. Performability evaluation of water distribution systems during maintenance procedures. IEEE Transactions on Systems, Man, and Cybernetics: Systems PP (2018), 1-17. DOI : https://doi. org/10.1109/TSMC.2017.2783188

C. F. Colebrook. 1939. Turbulent flow in pipes, with particular reference to the transition region between smooth and rough pipe laws. Journal of the Institution of Civil Engineers 11, 4 (February 1939), 133-156.

Paul Deane, M. Ó. Ciaráin, and Brian Ó. Gallachoir. 2017. An integrated gas and electricity model of the EU energy system to examine supply interruptions. Applied Energy 193 (2017), 479-490. DOI : https://doi.org/10.1016/j.apenergy.2017.02.039

Özge Dilaver, Zafer Dilaver, and Lester C. Hunt. 2014. What drives natural gas consumption in Europe? Analysis and projections. Journal of Natural Gas Science and Engineering 19 (2014), 125-136. DOI : https://doi.org/10.1016/j.jngse.2014. 04.002

Simona Esposito, Sonia Giovinazzi, Ludovica Elefante, and Iunio Iervolino. 2013. Performance of the L'Aquila (central Italy) gas distribution network in the $2009\left(M_{w}\right.$ 6.3) earthquake. Bulletin of Earthquake Engineering 11, 6 (2013), $2447-2466$. DOI : https://doi.org/10.1007/s10518-013-9478-8

Alberto Herrán González, Jesús M. de la Cruz, Bonifacio De Andrés-Toro, and José Luis Risco-Martín. 2009. Modeling and simulation of a gas distribution pipeline network. Applied Mathematical Modelling 33, 3 (2009), 1584-1600. DOI : https:// doi.org/10.1016/j.apm.2008.02.012

Scottish Government. 2014. Government On-Line Sustainability Performance Information Exchange of the Scottish Government. Retrieved on October 1, 2014 from http://data.glasgow.gov.uk/dataset/atlantic-quay-half-hourlygas-consumption.

Arild Helseth and Arne T. Holen. 2006. Reliability modeling of gas and electric power distribution systems; similarities and differences. In International Conference on Probabilistic Methods Applied to Power Systems 1-5. DOI : https://doi.org/10. 1109/PMAPS.2006.360422

Daniel Homm and Reinhard German. 2016. Analysis of hierarchical semi-Markov processes with parallel regions. In International GI/ITG Conference on Measurement, Modelling, and Evaluation of Computing Systems and Dependability and Fault Tolerance. Springer, 92-106. DOI : https://doi.org/10.1007/978-3-319-31559-1_9

András Horváth, Marco Paolieri, Lorenzo Ridi, and Enrico Vicario. 2012. Transient analysis of non-Markovian models using stochastic state classes. Performance Evaluation 69, 7-8 (2012), 315-335. DOI : https://doi.org/10.1016/j.peva.2011.11.002

Andras Horvath, Antonio Puliafito, Marco Scarpa, and Miklos Telek. 2000. Analysis and evaluation of non-Markovian stochastic Petri nets. In International Conference on Computer Performance Evaluation 171-187. DOI: https://doi.org/10. 1007/3-540-46429-8_13

Heiko Koziolek. 2010. Performance evaluation of component-based software systems: A survey. Performance Evaluation 67, 8 (2010), 634-658. DOI : https://doi.org/10.1016/j.peva.2009.07.007

Tao Li, M. Eremia, and M. Shahidehpour. 2008. Interdependency of natural gas network and power system security. IEEE Transactions on Power Systems 23, 4 (2008), 1817-1824. DOI : https://doi.org/10.1109/TPWRS.2008.2004739

Xiang Li, Emre Armagan, Asgeir Tomasgard, and Paul I. Barton. 2011. Stochastic pooling problem for natural gas production network design and operation under uncertainty. AIChE fournal 57, 8 (2011), 2120-2135. DOI : https://doi.org/10.1002/ aic.12419

Christoph Lindemann and Axel Thümmler. 1999. Transient analysis of deterministic and stochastic Petri nets with concurrent deterministic transitions. Performance Evaluation 36-37, 1-4 (1999), 35-54. DOI: https://doi.org/10.1016/ S0166-5316(99)00020-6

Christoph Lindemann, Axel Thümmler, Alexander Klemm, Marco Lohmann, and Oliver P. Waldhorst. 2002. Performance analysis of time-enhanced UML diagrams based on stochastic processes. In International Workshop on SW and Performance. ACM, 25-34. DOI : https://doi.org/10.1145/584369.584375

Francesco Longo and Marco Scarpa. 2009. Applying symbolic techniques to the representation of non-Markovian models with continuous PH distributions. In Performance Engineering Workshop. Springer, 44-58. DOI : https://doi.org/10.1007/ 978-3-642-02924-0_4

Noe Lopez-Benitez and Kishor S. Trivedi. 1993. Multiprocessor performability analysis. IEEE Transactions on Reliability 42 , 4 (December 1993), 579-587. DOI : https://doi.org/10.1109/24.273586 
John Lygeros and Maria Prandini. 2010. Stochastic hybrid systems: A powerful framework for complex, large scale applications. European fournal of Control 16, 6 (2010), 583-594. DOI : https://doi.org/doi.org/10.3166/ejc.16.583-594

Tom Mens and Pieter Van Gorp. 2006. A taxonomy of model transformation. Electronic Notes in Theoretical Computer Science 152 (2006), 125-142. DOI : https://doi.org/10.1016/j.entcs.2005.10.021

Object Management Group. 2009. UML Profile for MARTE: Modeling and Analysis of Real-Time Embedded Systems v1.0. Object Management Group.

Object Management Group. 2017. OMG System Modeling Language. Object Management Group.

ORIS Tool. 2018. Homepage. Retrieved on October 1, 2018 from http://www.oris-tool.org.

Willem Pino, Daniel Worm, Ruud van der Linden, and Robert Kooij. 2016. The reliability of a gas distribution network: A case study. In International Conference on System Reliability and Science. 122-129. DOI : https://doi.org/10.1109/ICSRS. 2016.7815850

Pavel Praks, Josef Chudoba, Radim Bris, and Miroslav Koucky. 2007. Reliability analysis of a natural gas compression station and surrounding gas pipeline network with assuming of performance changes by a dispatcher. Risk, Reliability and Societal Safety 3 (2007), 2323-2330.

Pavel Praks, Sergio Contini, and Vytis Kopustinskas. 2014. Monte Carlo and fault tree approaches in reliability applications of gas transmission network. In International Scientific Conference on Electric Power Engineering. 69-74. DOI : https://doi. org/10.1109/EPE.2014.6839421

Meysam Qadrdan, Jianzhong Wu, Nick Jenkins, and Janaka Ekanayake. 2014. Operating strategies for a GB integrated gas and electricity network considering the uncertainty in wind power forecasts. IEEE Transactions on Sustainable Energy 5, 1 (January 2014), 128-138. DOI : https://doi.org/10.1109/TSTE.2013.2274818

Božidar Soldo. 2012. Forecasting natural gas consumption. Applied Energy 92 (2012), 26-37. DOI : https://doi.org/10.1016/j. apenergy.2011.11.003

Jolanta Szoplik. 2012. The Gas Transportation in a Pipeline Network, Advances in Natural Gas Technology. Dr. Hamid AlMegren (Ed.), InTech, 339-359.

Jolanta Szoplik. 2015. Forecasting of natural gas consumption with artificial neural networks. Energy 85 (2015), 208-220 DOI : https://doi.org/10.1016/j.energy.2015.03.084

Tiedo Tinga. 2012. Mechanism Based Failure Analysis. Netherlands Defence Academy, Kraanstraat.

Mirco Tribastone and Stephen Gilmore. 2008. Automatic translation of UML sequence diagrams into PEPA models. In International Conference on Quantitative Evaluation of Systems. IEEE, 205-214. DOI : https://doi.org/10.1109/QEST.2008. 18

Chiara Vianello and Giuseppe Maschio. 2014. Quantitative risk assessment of the Italian gas distribution network. fournal of Loss Prevention in the Process Industries 32, 0 (2014), 5-17. DOI : https://doi.org/10.1016/j.jlp.2014.07.004

Enrico Vicario, Luigi Sassoli, and Laura Carnevali. 2009. Using stochastic state classes in quantitative evaluation of densetime reactive systems. IEEE Transactions on Software Engineering 35, 5 (2009), 703-719. DOI : https://doi.org/10.1109/TSE. 2009.36

Dazhi Wang and Kishor S. Trivedi. 2007. Reliability analysis of phased-mission system with independent component repairs. IEEE Transactions on Reliability 56, 3 (2007), 540-551. DOI : https://doi.org/10.1109/TR.2007.903268

Hui Wang and Ian J. Duncan. 2014. Likelihood, causes, and consequences of focused leakage and rupture of U.S. natural gas transmission pipelines. Journal of Loss Prevention in the Process Industries 30 (2014), 177-187. DOI : https://doi.org/ 10.1016/j.jlp.2014.05.009

Wolfram Mathematica 2017. Wolfram Mathematica 11. Wolfram Research, Oxfordshire, United Kingdom.

Victor M. Zavala. 2014. Stochastic optimal control model for natural gas networks. Computers \& Chemical Engineering 64 (2014), 103-113. DOI : https://doi.org/10.1016/j.compchemeng.2014.02.002

Received June 2017; revised October 2018; accepted October 2018 